\title{
Sustained activation of SMAD3/SMAD4 by FOXM1 promotes TGF- $\beta$-dependent cancer metastasis
}

\author{
Jianfei Xue, ${ }^{1}$ Xia Lin, ${ }^{2}$ Wen-Tai Chiu, ${ }^{1}$ Yao-Hui Chen, ${ }^{1}$ Guanzhen Yu, ${ }^{1}$ Mingguang Liu, ${ }^{1}$ \\ Xin-Hua Feng, ${ }^{2,3}$ Raymond Sawaya, ${ }^{1}$ René H. Medema, ${ }^{4}$ Mien-Chie Hung, 5,6,7 and Suyun Huang ${ }^{1,6}$ \\ ${ }^{1}$ Department of Neurosurgery, The University of Texas M.D. Anderson Cancer Center, Houston, Texas, USA. ${ }^{2}$ Michael E. DeBakey Department of Surgery and \\ ${ }^{3}$ Department of Molecular and Cellular Biology, Baylor College of Medicine, Houston, Texas, USA. ${ }^{4}$ Department of Medical Oncology, \\ University Medical Center Utrecht, Utrecht, The Netherlands. ${ }^{5}$ Department of Molecular and Cellular Oncology, \\ The University of Texas M.D. Anderson Cancer Center, Houston, Texas, USA. ${ }^{6}$ Program in Cancer Biology, \\ The University of Texas Graduate School of Biomedical Sciences at Houston, Houston, Texas, USA. \\ ${ }^{7}$ Graduate Institute of Cancer Biology and Center for Molecular Medicine, China Medical University, Taichung, Taiwan.
}

\begin{abstract}
A key feature of TGF- $\beta$ signaling activation in cancer cells is the sustained activation of SMAD complexes in the nucleus; however, the drivers of SMAD activation are poorly defined. Here, using human and mouse breast cancer cell lines, we found that oncogene forkhead box M1 (FOXM1) interacts with SMAD3 to sustain activation of the SMAD3/SMAD4 complex in the nucleus. FOXM1 prevented the E3 ubiquitin-protein ligase transcriptional intermediary factor $1 \gamma$ (TIF1 $\gamma$ ) from binding SMAD3 and monoubiquitinating SMAD4, which stabilized the SMAD3/SMAD4 complex. Loss of FOXM1 abolished TGF- $\beta$-induced SMAD3/SMAD4 formation. Moreover, the interaction of FOXM1 and SMAD3 promoted TGF- $\beta / S M A D 3-$ mediated transcriptional activity and target gene expression. We found that FOXM1/SMAD3 interaction was required for TGF- $\beta$-induced breast cancer invasion, which was the result of SMAD3/SMAD4-dependent upregulation of the transcription factor SLUG. Importantly, the function of FOXM1 in TGF- $\beta$-induced invasion was not dependent on FOXM1's transcriptional activity. Knockdown of SMAD3 diminished FOXM1-induced metastasis. Furthermore, FOXM1 levels correlated with activated TGF- $\beta$ signaling and metastasis in human breast cancer specimens. Together, our data indicate that FOXM1 promotes breast cancer metastasis by increasing nuclear retention of SMAD3 and identify crosstalk between FOXM1 and TGF- $\beta$ /SMAD3 pathways. This study highlights the critical interaction of FOXM1 and SMAD3 for controlling TGF- $\beta$ signaling during metastasis.
\end{abstract}

\section{Introduction}

The TGF- $\beta$ signaling pathway plays pivotal roles in diverse developmental processes and the pathogenesis of many diseases, including cancer $(1,2)$. The TGF- $\beta$ signaling cascade is initiated by binding of the TGF- $\beta$ ligand to pairs of receptor serine/threonine kinases known as type I and type II receptors. Upon binding of TGF- $\beta$, type II receptors phosphorylate and activate type I receptors. The activated type I receptors phosphorylate intracellular effectors SMAD2/SMAD3, which form a complex with SMAD4 and then shuttle to the nucleus (2). A key feature of TGF- $\beta$ signaling activation is that the SMAD2 or SMAD3 proteins in activated SMAD4SMAD2/SMAD3 complexes in the nucleus bind other DNA-binding transcription factors as partners for target gene recognition and transcriptional regulation $(3,4)$. Furthermore, it is generally accepted that the formation of the SMAD2/SMAD4 or SMAD3/ SMAD4 complex is necessary for the nuclear retention of SMAD2 or SMAD3 and thus for activation of TGF- $\beta$ signaling (5-7).

The TGF- $\beta$ signaling pathway, particularly regarding the transcriptional activation of the SMADs complex, is tightly controlled by specialized inhibitory factors. For example, in normal cells, transcriptional intermediary factor $1 \gamma$ (TIF $1 \gamma)$ controls SMAD2/ SMAD3 activity by regulating SMAD4 monoubiquitination, which leads to the disassembly of the SMADs transcriptional complex and

Conflict of interest: The authors have declared that no conflict of interest exists. Citation for this article: J Clin Invest. 2014;124(2):564-579. doi:10.1172/JCI71104. a forced exit of SMAD2/SMAD3 from the nucleus $(8,9)$. However, in cancer cells, the SMADs transcriptional complex remains in the nucleus. The molecular mechanisms regulating this maintenance of the SMAD transcriptional complex remain elusive.

The major cause of death from cancer is due to metastasis, the control or prevention of which remains a challenge in cancer research. The TGF- $\beta$ signaling pathway is aberrantly activated in human cancers and plays a critical role in cancer progression and metastasis in some tumor cells (2). For example, in breast cancer, TGF- $\beta$ stimulates proliferation, epithelial-mesenchymal transition (EMT), invasion, and metastasis (4). Previous studies have reported that inactivating germline alterations in SMAD3 and SMAD4 are rare (10), whereas aberrant expression of SMAD3 and SMAD4 is more common in breast cancer (10). Specifically, previous studies have found that P-SMAD3 (an activated SMAD3) and SMAD4 were expressed in $65 \%$ to $74.0 \%$ and $61.0 \%$ to $92 \%$ of all breast cancer specimens, respectively (10-13), and that the expression of SMAD3 and SMAD4 is ubiquitous in human infiltrating ductal breast carcinomas (14). Moreover, SMAD3 and SMAD4 mRNA expression levels were significantly elevated in human breast carcinomas relative to levels in surrounding unaffected tissues (10). Furthermore, it has been shown that the levels of SMAD3 activation induced by TGF- $\beta$ in breast cancer are directly correlated with lymph node metastasis or metastasis to other organ systems $(13,15)$.

Like TGF- $\beta$ /SMAD3 signaling, the forkhead box M1 (FOXM1) transcriptional factor is aberrantly activated in most human can- 
cers (16) and is a key regulator of cancer pathogenesis (17-19). Our previous results have demonstrated that FOXM1 plays a critical role in the tumorigenesis of brain tumor stem cells (20). In normal breast tissue, FOXM1 expression is often absent or weaker than in breast cancer, and FOXM1 may only be expressed in luminal epithelial progenitors $(21,22)$. In breast cancer, the levels of FOXM1 correlate positively with the tumor grade $(21,23)$, which is associated with poor prognosis (24). However, the roles of FOXM1 in promoting breast cancer metastasis and its underlying mechanism have not been elucidated.

Given that both FOXM1 and TGF- $\beta /$ SMAD 3 are activated in many cancers, including breast cancer, we investigated potential crosstalk between these 2 intensively studied oncogenic pathways. We explored the possibility that FOXM1 is a key component in regulating nuclear retention of the SMAD3/SMAD4 complex in TGF- $\beta$ signaling and determined the biological consequences of FOXM1-SMAD3/SMAD4 interaction in breast cancer metastasis.

\section{Results}

FOXM1 regulates TGF- $\beta / S M A D 3$-induced transcriptional activity and gene expression. First, we examined FOXM1 expression in a panel of human and mouse breast cancer cell lines. We found that the metastatic breast cancer cell lines expressed higher levels of FOXM1 than the nonmetastatic breast cancer cell lines (Figure 1A) and FOXM1B was the predominant isoform expressed in the human breast cancer cell lines (Supplemental Figure 1A; supplemental material available online with this article; doi:10.1172/JCI71104DS1). Next, we investigated whether FOXM1 modulates TGF- $\beta$ signaling, as it is well known that TGF- $\beta$ signaling has a pivotal role in breast cancer metastasis (4). Also, the expression level of nuclear SMAD3, a key signaling molecule for TGF- $\beta$ signaling, is positively correlated with the expression level of nuclear FOXM1 in the above breast cancer cell lines (Figure 1A), although the expression levels of FOXM1, total SMAD3, and SMAD4 are not correlated (Supplemental Figure 1B). We produced FOXM1 overexpression in 4T07 and BT-474 cells by transfection of FOXM1 expression plasmid into the cell lines and established 2 stable FOXM1 transfectants from each cell line (Figure 1B). We stably knocked down FOXM1 in 4T1 and MDA-MB-231 cells by using 2 different shRNAs targeting FOXM1 (Figure 1B). Then, the effect of altered expression of FOXM1 on TGF- $\beta 1$ target genes was examined in the above cells. Altered expression of FOXM1 did not change the expression levels of SMAD3 and SMAD4 (Supplemental Figure 1C). However, knockdown of FOXM1 in MDA-MB-231 cells inhibited the TGF- $\beta 1$-induced expression of PAI- 1 , JunB, and Smurf1, the prototypic TGF- $\beta$ signaling target genes (Figure $1 \mathrm{C}$ ), whereas overexpression of FOXM1 in 4T07 and BT-474 cells increased the TGF- $\beta 1$-induced expression of these genes (Supplemental Figure 1D). Moreover, deletion of FOXM1 in immortalized Foxm $1^{f / f l}$ mouse embryonic fibroblasts (MEFs) abolished these multiple TGF- $\beta 1$-induced gene responses (Figure $1 D$ ). These results suggest that FOXM1 enhances TGF- $\beta$-induced gene expression.

As SMAD2 and SMAD3 are major moderators for TGF- $\beta$ signaling, we tested the role of FOXM1 in SMAD2/SMAD3-mediated transcriptional activity. Knockdown of FOXM1 by shFOXM1 in MDA-MB-231 cells resulted in a decrease in the activity of SBE4-Luc, a SMAD3-specific luciferase reporter construct (Figure 1E). Reexpression of FOXM1 by using shRNA-resistant FOXM1 (FOXM1shR) rescued the inhibitory effect of FOXM1 shRNA on SBE4Luc activity (Figure 1E), suggesting a specific FOXM1 function in TGF- $\beta 1 /$ SMAD3 signaling. Similar results were also observed in FOXM1 gene deletion cells. Deletion of FOXM1 in Foxm $1^{f / f l}$ MEFs abolished the effect of TGF- $\beta 1$ on activation of SBE4-Luc, whereas reexpression of FOXM1 rescued the inhibitory effect of FOXM1 deletion in SBE4-Luc activity (Figure 1F). In addition, increased FOXM1 expression did not enhance the activity of ARELuc, a TGF- $\beta 1$-responsive but SMAD2-specific luciferase reporter (Supplemental Figure 1E), suggesting that the role for FOXM1 in TGF- $\beta$ signaling is specifically through the regulation of SMAD3.

Next, the mechanisms by which FOXM1 regulates TGF- $\beta$ signaling were investigated in $\mathrm{HaCaT}$ cells, a TGF- $\beta 1$-sensitive cell line widely used for TGF- $\beta$ research. TGF- $\beta 1$ induced the expression of PAI-1 in HaCaT cells (Figure 1G). Overexpression of FOXM1 resulted in the upregulation of TGF- $\beta 1$-induced $P A I-1$ expression but did not affect total levels of SMAD3 or TGF- $\beta 1$ type I and II receptors (T $\beta R I$ and T $\beta R I I)$ (Figure $1 G)$. Also, overexpression of FOXM1 did not affect the PPM1A-mediated dephosphorylation of activated SMAD3 (6), which was phosphorylated by a constitutively active mutant form of T $\beta$ RI (ALK5-T202D) (Supplemental Figure $1 F)$. These results suggest that FOXM1 takes action after SMAD3 activation and before SMAD3 inactivation by dephosphorylation.

We further examined the effect of FOXM1 on the expression of endogenous TGF- $\beta 1$-dependent genes. TGF- $\beta 1$ induced the expression of mRNAs for PAI- 1 and SMAD7, the prototypic TGF- $\beta 1$ signaling target genes, and overexpression of FOXM1 enhanced this effect (Figure 1, H and I). These results indicate that FOXM1 regulates TGF- $\beta 1 /$ SMAD3-mediated transcription.

FOXM1 directly interacts with SMAD3. We examined whether FOXM1 regulates TGF- $\beta$ signaling via a direct interaction with SMAD3. In 293T cells cotransfected with a HA-tagged FOXM1 expression vector and various Flag-tagged SMAD expression vectors, FOXM1 was coprecipitated with SMAD3, but not with SMAD1, SMAD2, SMAD4, SMAD5, or SMAD6 (Figure 2A). Also, the in vitro interactions between FOXM1 and SMAD3 were analyzed using recombinant GST-SMAD3 and His-FOXM1. In a GST pulldown assay, purified His-FOXM1 bound to GST-SMAD3 but not to the GST control (Figure 2B), indicating a direct interaction between SMAD3 and FOXM1.

By using SMAD3 deletion mutants, we found that the interaction between SMAD3 and FOXM1 was mediated by the MH2 domain of SMAD3 (Figure 2C). Deletion of the $\mathrm{C}$ terminal did not change the binding affinity of FOXM1 for SMAD3, but deletion of the $\mathrm{N}$ terminus abolished this binding (Figure 2D). Thus, the $N$-terminal domain of FOXM1 mediates its interaction with SMAD3.

In addition to the above transfection experiments, we also determined the interaction of endogenous FOXM1 and SMAD3 at the physiological level. As shown in Figure 2E, endogenous FOXM1 associated with SMAD3 in HaCaT cells, as shown by coimmunoprecipitation assay, and this association was increased in response to TGF- $\beta 1$ treatment (Figure $2 \mathrm{E}$ ). The interaction between endogenous FOXM1 and SMAD3 was also detected in both 4T07 and 4T1 breast cancer cells, and the association was increased with overexpression of FOXM1 but decreased with knockdown of FOXM1 (Supplemental Figure 2).

FOXM1 increases SMAD3 nuclear retention through maintenance of the SMAD3/SMAD4 complex. Nuclear localization of SMAD3 and its direct binding to target gene promoters are necessary for TGF- $\beta 1$ signaling. To understand the molecular mechanism of FOXM1 regulation on SMAD3 activity, we first determined whether FOXM1 might modify the nuclear translocation of SMAD3. The location of SMAD3 in the cytoplasmic and nuclear fractions of TGF- $\beta 1-$ 
A

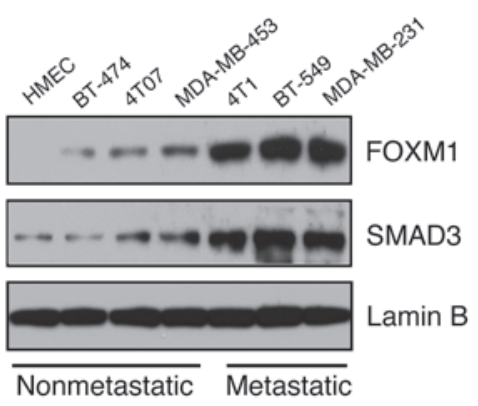

B

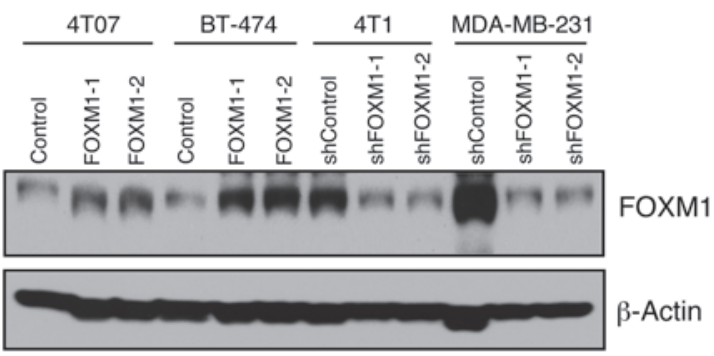

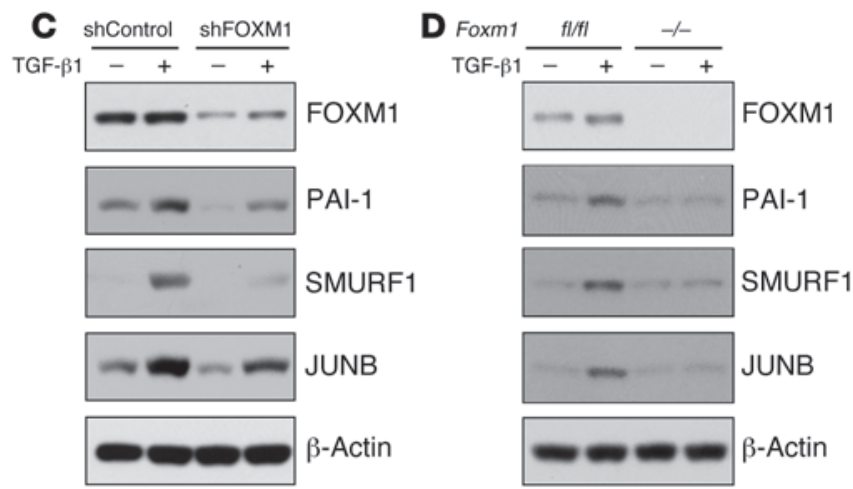

E

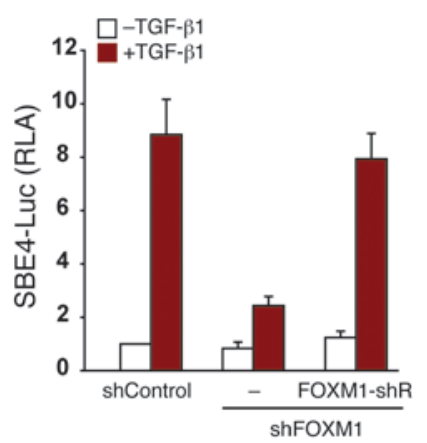

$\mathbf{F}$

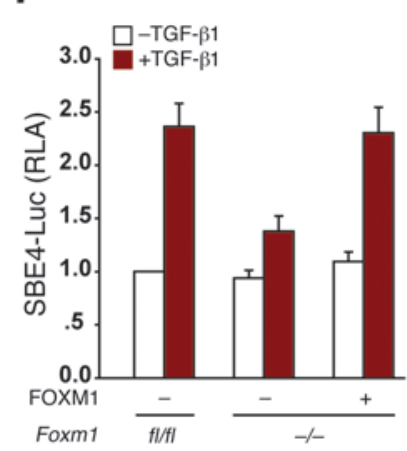

G

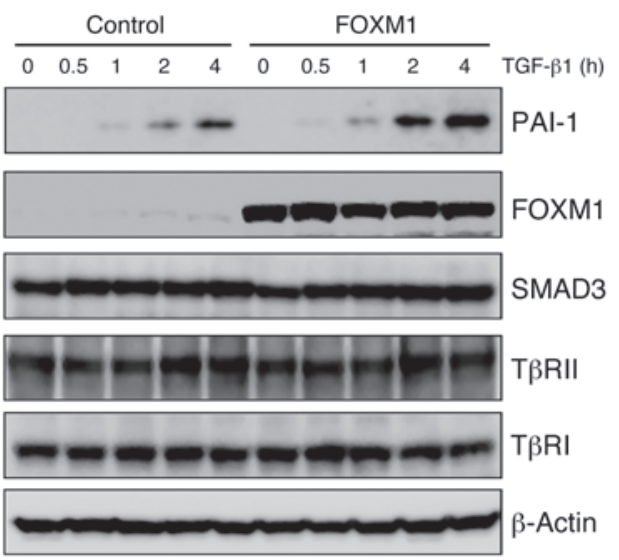

H

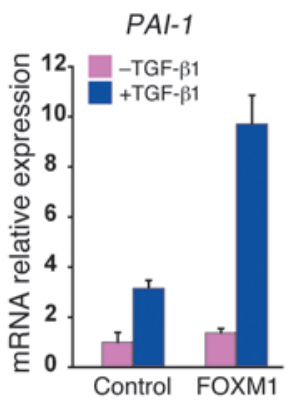

I

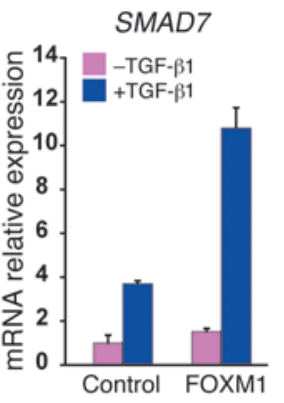

Figure 1

FOXM1 regulates TGF- $\beta$ /SMAD3-induced transcriptional activity and gene expression. (A) Western blotting of nuclear SMAD3 and FOXM1 proteins in 7 cell lines. (B) FOXM1 protein expression was analyzed by immunoblotting. (C) MDA-MB-231 cells stably expressing control shRNA or FOXM1 shRNA were incubated with or without TGF- $\beta 1$ ( $5 \mathrm{ng} / \mathrm{ml})$ for 2 hours. (D) Foxm $1^{\text {flfl }}$ MEFs were transduced with 500 MOI of Adeno-Cre virus to delete FOXM1. Both the Cre-transduced MEFs (Foxm $1^{-1}$ ) and control virus-transduced MEFs (Foxm $1^{\text {fllff}}$ ) were then treated with TGF- $\beta 1$ $(5 \mathrm{ng} / \mathrm{ml})$ for 2 hours. (E) Luciferase activity was measured 48 hours after incubation of cells with or without TGF- $\beta 1$ ( $5 \mathrm{ng} / \mathrm{ml})$ for 20 hours by the dual luciferase assay. Values are mean \pm SD for triplicate samples. RLA, relative luciferase activity. (F) Deletion of Foxm1 abolished SMAD3activated transcription activity. (G) HaCaT cells were transfected with empty vector or the Flag-FOXM1 vector, treated with TGF- $\beta 1$, and analyzed by immunoblotting using appropriate antibodies as indicated. (H and I) FOXM1 enhanced TGF- $\beta 1$-induced PAI-1 and SMAD7 gene expression by qRT-PCR. HaCaT cells transfected with control vector or Flag-FOXM1 in the presence or absence of TGF- $\beta 1$ ( $5 \mathrm{ng} / \mathrm{ml}$ ) for $2 \mathrm{hours}$.

treated $\mathrm{HaCaT}$ cells or $4 \mathrm{~T} 07$ breast cancer cells was examined in the presence of T $\beta$ RI inhibitor SB431542, which blocks newly formed SMAD3 phosphorylation and nuclear translocation. By using Western blotting in $\mathrm{HaCaT}$ cells and immunofluorescence staining in 4T07 cells, we found that TGF- $\beta 1$ treatment significantly increased the nuclear localization of SMAD3 (Figure 3, A and B). SB431542 treatment increased the level of cytoplasmic SMAD3 and decreased the level of nuclear SMAD3 in control cells (Figure 3, $\mathrm{A}$ and $\mathrm{B})$. In contrast, overexpression of FOXM1 enhanced the nuclear localization of SMAD3 (Figure 3, A and B).

To further confirm that FOXM1 increases SMAD3 nuclear retention, we carried out an interspecies heterokaryon assay by fusing human HaCaT stable cells, expressing FOXM1 and transfected with Flag-SMAD3, with mouse NIH3T3 cells. As shown 
A
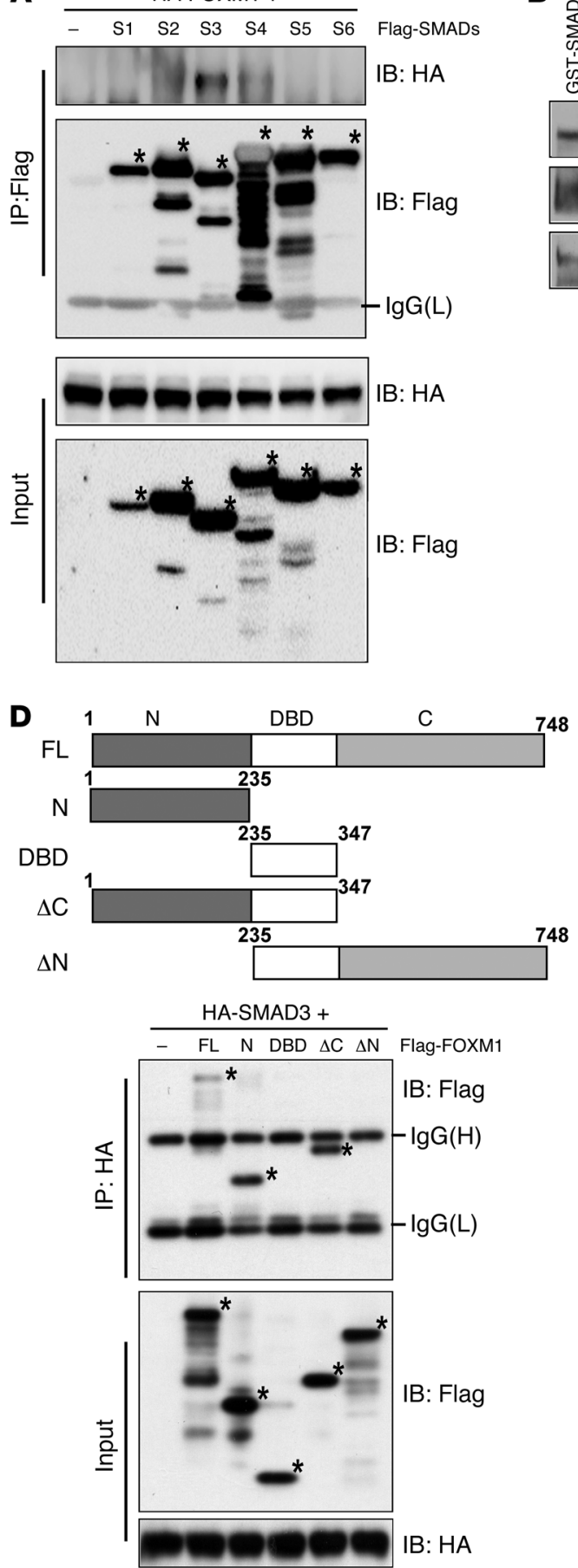

B

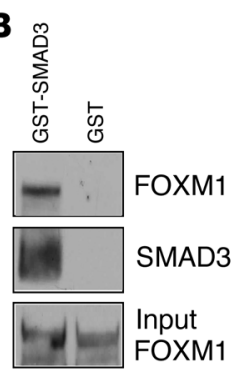

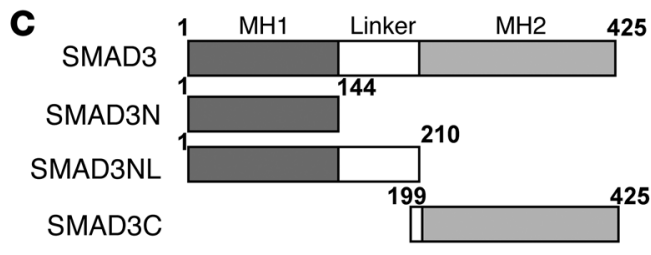
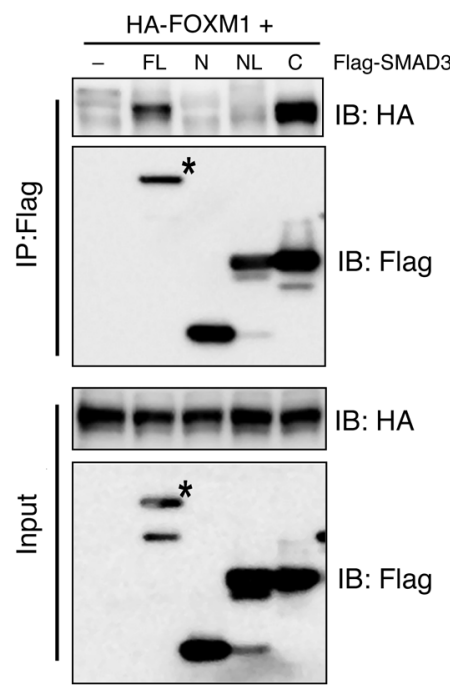

E $\begin{array}{cccc}\text { Input } & \text { IP: } & \text { IP: } \\ n n-\beta 1 & \frac{\text { IgG }}{-} & \frac{\text { SMAD3 }}{-}+\end{array}$

IB: FOXM1

IB: SMAD3
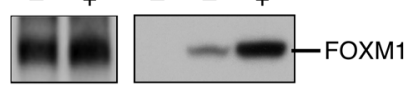

IB: p-SMAD3
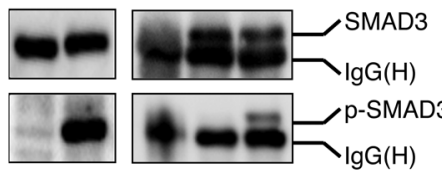

\section{Figure 2}

FOXM1 interacts with SMAD3 directly. (A) Flag-SMADs and HA-FOXM1 were expressed in 293T cells. Each Flag-SMAD protein was immunoprecipitated using an anti-Flag antibody. Asterisks indicate Flag-SMAD expression. (B) GST pulldown was performed using purified GST-SMAD3 and 6xHis-FOXM1, followed by immunoblotting with anti-FOXM1 and anti-SMAD3 antibodies. (C) The MH2 domain of SMAD3 was involved in the interaction with FOXM1. Upper panel: Schematic illustration of SMAD3 deletion mutants. The numbers indicate the amino acid positions. Lower panel: Flag-SMAD3 deletion mutants were coexpressed with HA-FOXM1 in 293T cells. Asterisks indicate full-length Flag-SMAD3 expression. (D) The $N$-terminal domain of FOXM1 was involved in the interaction with SMAD3. Upper panel: schematic illustration of FOXM1 deletion mutants. Lower panel: Flag-FOXM1 deletion mutants were coexpressed with HA-SMAD3 in 293T cells. The cells were subjected to immunoprecipitation with an HA antibody. Asterisks indicate different fragments of Flag-FOXM1 expression. (E) Coimmunoprecipitation of endogenous FOXM1 with SMAD3 in HaCaT cells. Cells were treated with or without TGF- $\beta 1(5 \mathrm{ng} / \mathrm{ml})$ for 1 hour. 
A

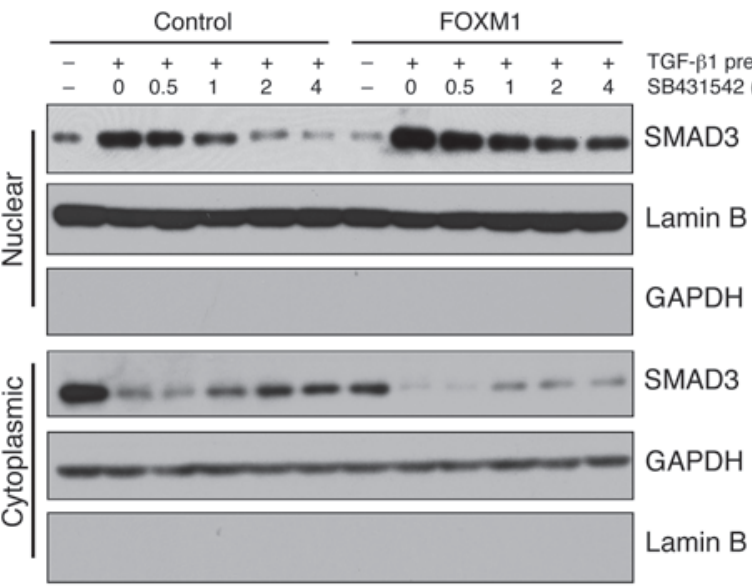

B

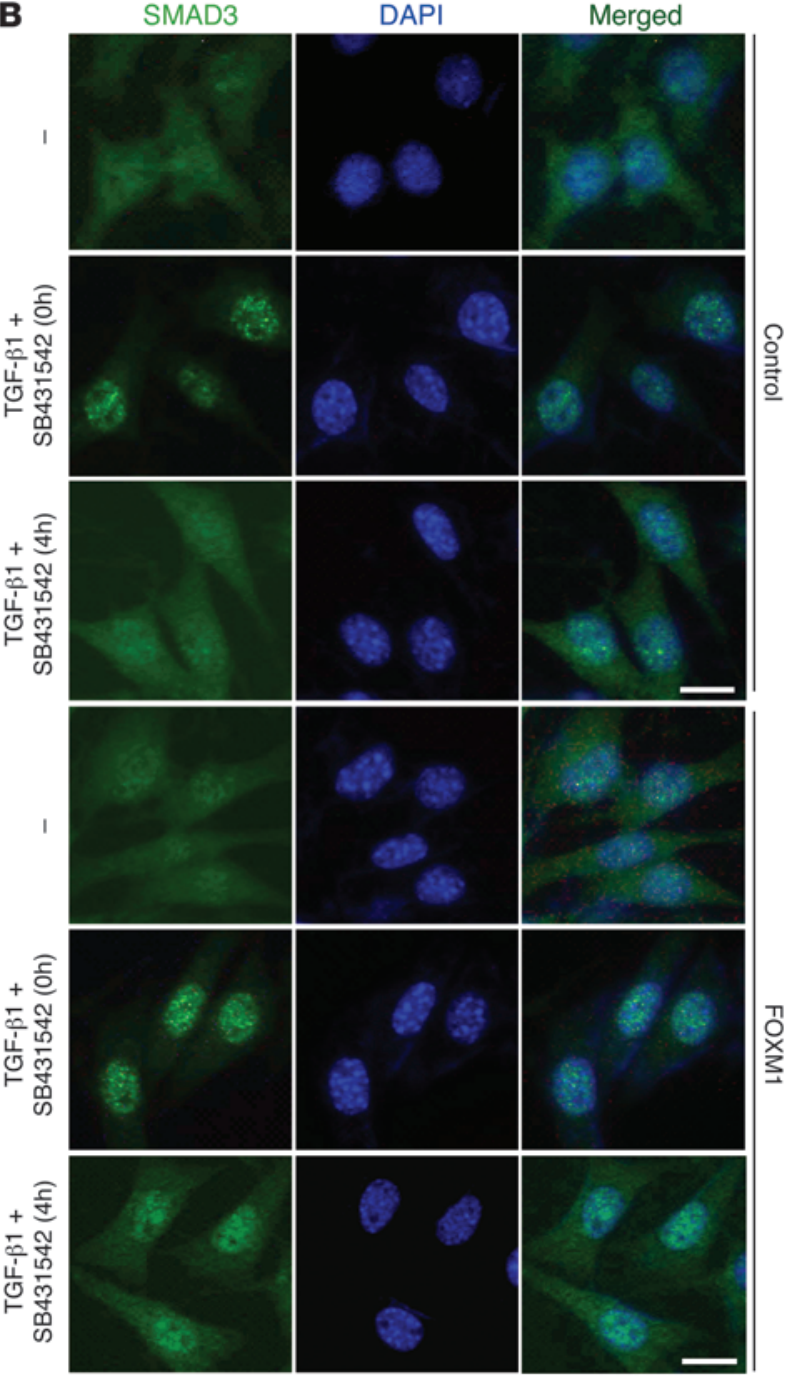

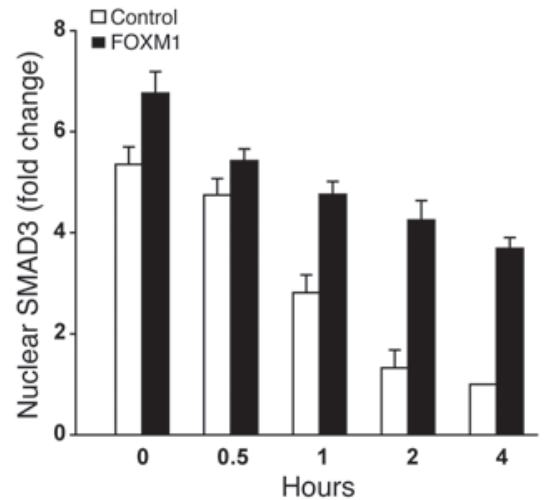

C
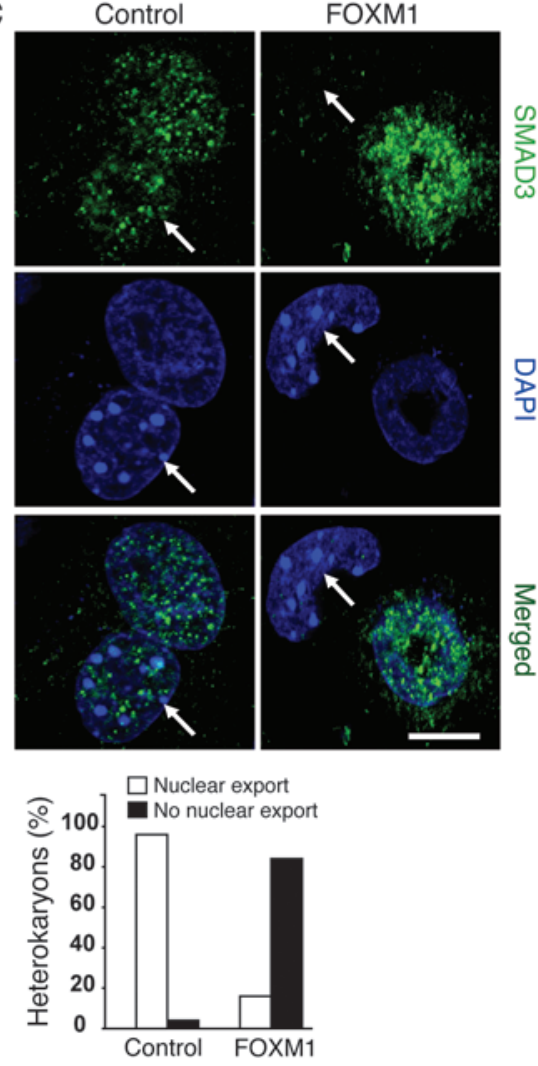

D

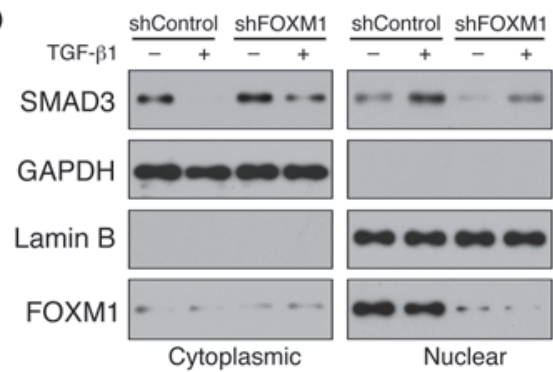




\section{Figure 3}

FOXM1 increases nuclear retention of SMAD3. (A) FOXM1 increased nuclear retention of SMAD3. HaCaT cells transfected with FlagFOXM1 were treated with TGF- $\beta 1(5 \mathrm{ng} / \mathrm{ml})$ for 30 minutes; cells were washed 3 times to remove TGF- $\beta 1$ and treated with SB431542 for up to 4 hours. Cells were harvested at indicated times, and both the nuclear and cytoplasmic fractions were collected. Values are mean \pm SD from 2 experiments. (B) FOXM1 increased nuclear retention of SMAD3. 4T07 control and FOXM1 overexpression cells were treated with TGF- $\beta 1(5 \mathrm{ng} / \mathrm{ml})$ for 30 minutes; cells were washed 3 times to remove TGF- $\beta 1$ and treated with SB431542 for 4 hours. Intensity of nuclear SMAD3 among these cells was quantified using NIH ImageJ software. The percentages of nuclear SMAD3 level shown at the right represent the mean of 3 independent experiments, and error bars indicate the SEM. Scale bars: $20 \mu \mathrm{m}$. (C) SMAD3 nuclear export as determined by a heterokaryon assay. HaCaT stable cells expressing FOXM1 were transfected with Flag-SMAD3 plasmids, and the cells were fused with murine NIH 3 T3 cells. NIH 3 T3 nuclei that are easily distinguished by the presence of brightly stained blocks in heterorkaryons are marked by arrows. Scale bars: $10 \mu \mathrm{m}$. Relocalization of SMAD3 to the mouse nucleus was rarely seen in heterokaryons expressing FOXM1, as shown with the percentages of heterokaryons in right panel. (D) Knockdown of FOXM1 reduced the level of nuclear SMAD3. MDA-MB-231 cells stably expressing shControl or shFOXM1 were treated with TGF- $\beta 1(5 \mathrm{ng} / \mathrm{ml})$ for 1 hour.

in Figure 3C, we detected the relocalization of SMAD3 from the human nucleus to the mouse cell nucleus in a significant portion of heterokaryons in the control group. In contrast, in the majority of heterokaryons overexpressing FOXM1, SMAD3 remained in the human nucleus, suggesting that FOXM1 increases SMAD3 nuclear retention (Figure 3C).

FOXM1-controlled SMAD3 nuclear retention was also observed in breast cancer cells. In untreated MDA-MB-231 cells, SMAD3 was localized both in the cytoplasm and nucleus. TGF- $\beta 1$ treatment significantly increased the nuclear localization of SMAD3 in these cells (Figure 3D). In contrast, knockdown of FOXM1 in MDA-MB-231 cells reduced the nuclear localization of SMAD3 (Figure 3D). Collectively, these data indicate that FOXM1 positively regulates TGF- $\beta 1$-induced SMAD3 nuclear localization.

To determine the mechanism by which FOXM1 enhances the nuclear retention of SMAD3, we first tested whether FOXM1 blocks the function of RanBP3, which recognizes the dephosphorylated form of SMAD3 and promotes its nuclear export (7). We found that endogenous FOXM1 did not interact with RanBP3 and had no effect on the inhibition of RanBP3 in TGF- $\beta 1$-induced SBE4-Luc reporter activity (data not shown). Next, we tested whether FOXM1 enhances the binding of SMAD3 to SMAD4, since the formation of the SMAD3/

\section{Figure 4}

SMAD4 complex is necessary for the nuclear retention of SMAD3 (5). Interestingly, we found that FOXM1 coexisted with SMAD3 and SMAD4 as a ternary complex and that the ternary complex formation was remarkably increased upon the TGF- $\beta$ signaling activation mediated by ALK5-T202D (Figure 4A). Moreover, overexpression of FOXM1 significantly increased the level of the SMAD3/SMAD4 complex induced by ALK5-T202D (Figure 4B).

FOXM1 is required for formation of the nuclear SMAD3/SMAD4 complex. To further determine whether FOXM1 is indispensable for formation of the nuclear SMAD3/SMAD4 complex, we used FOXM1 knockdown or knockout cells. Knockdown of FOXM1 decreased the level of the SMAD3/SMAD4 complex induced by TGF- $\beta 1$ in MDA-MB-231 cells (Figure 5A). Also, as visualized by immunofluorescence, SMAD3 and SMAD4 were colocalized in the nucleus when induced by TGF- $\beta 1$ in MDA-MB-231 cells, whereas knockdown of FOXM1 reduced their nuclear colocalization (Figure 5B), confirming that FOXM1 is essential for the sustained activation of the SMAD3/SMAD4 complex. Moreover, the SMAD3/SMAD4 complex formation was increased with overexpression of FOXM1 but decreased with knockdown of FOXM1 in both 4T07 and 4T1 breast cancer cells (Supplemental Figure 2). Furthermore, deletion of FOXM1 in Foxm $1^{f l f l}$ MEFs virtually abolished TGF- $\beta 1$-induced complex formation (Figure 5C) and SMAD3/SMAD4 nuclear colocalization (Figure 5D), further confirming that FOXM1 is required for formation of the nuclear SMAD3/SMAD4 complex.

FOXM1 attenuates the inhibitory effects of TIF1 $\gamma$ on TGF- $\beta$ signaling. To further explore the detailed mechanism(s) of how FOXM1 controls the duration of the SMAD3/SMAD4 complex conformation, we tested whether FOXM1 inhibits the function of nuclear corepressors that disrupt the SMAD3/SMAD4 complex in the nucleus, and hence the SMAD3-mediated transcription; the effects of SnoN/Ski (25), TGIF (26), and TIF1 $\gamma$ (9) were analyzed for this purpose. We found that these repressors exhibited robust inhibition on TGF- $\beta 1$ mediated responses in both 4T07 (Figure 6A) and HaCaT cells (Supplemental Figure 3A). However, overexpression of FOXM1 reduced

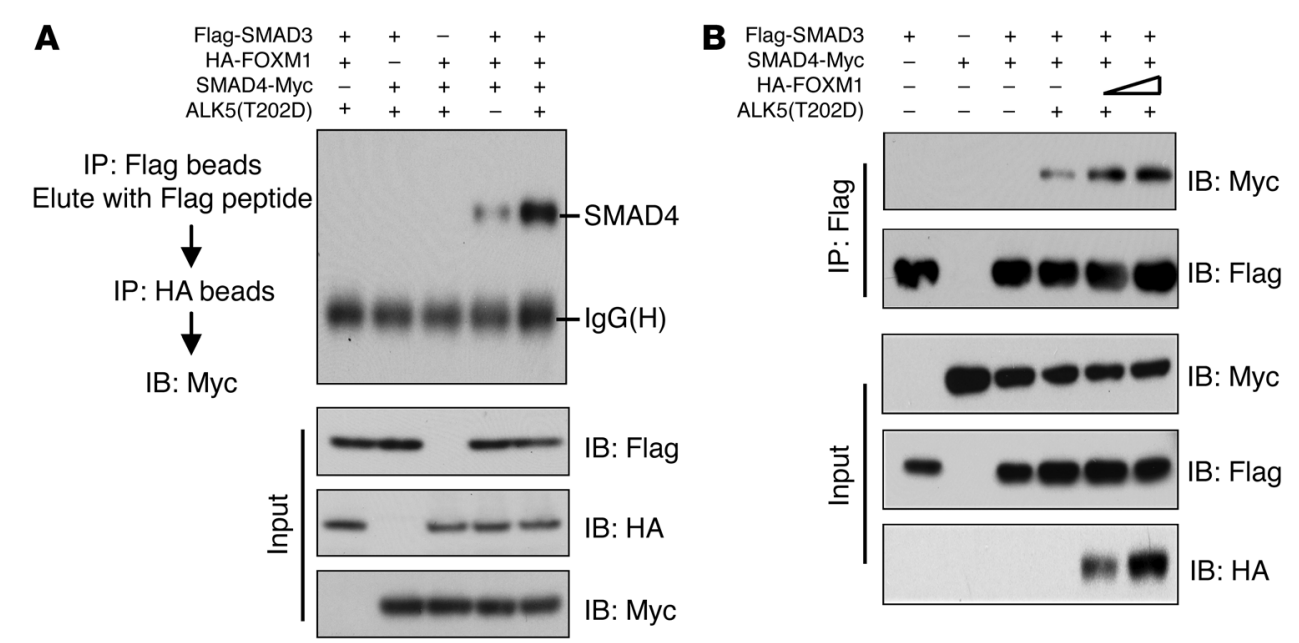

FOXM1 sustains the SMAD3/SMAD4 complex. (A) SMAD3, SMAD4, and FOXM1 formed a ternary complex. The indicated plasmids were cotransfected into 293T cells. The ternary complex was detected by sequential immunoprecipitation with Flag and HA beads, followed by immunoblot with the Myc antibody. (B) FOXM1 increased formation of the SMAD3/SMAD4 complex. 293T cells were transfected with the indicated plasmids. The amount of SMAD4 bound to SMAD3 was detected by immunoblot with an anti-Myc antibody (top). 
A
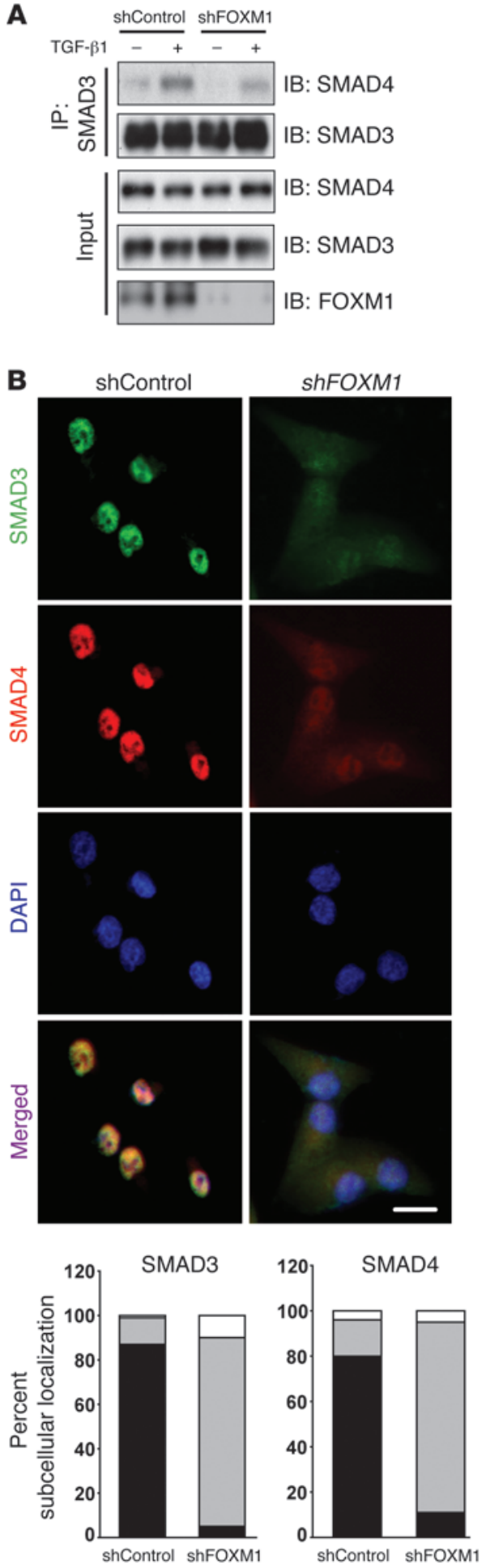

Nuclea

$\square$ Nuclear + cytoplasmic

$\square$ Cytoplasmic
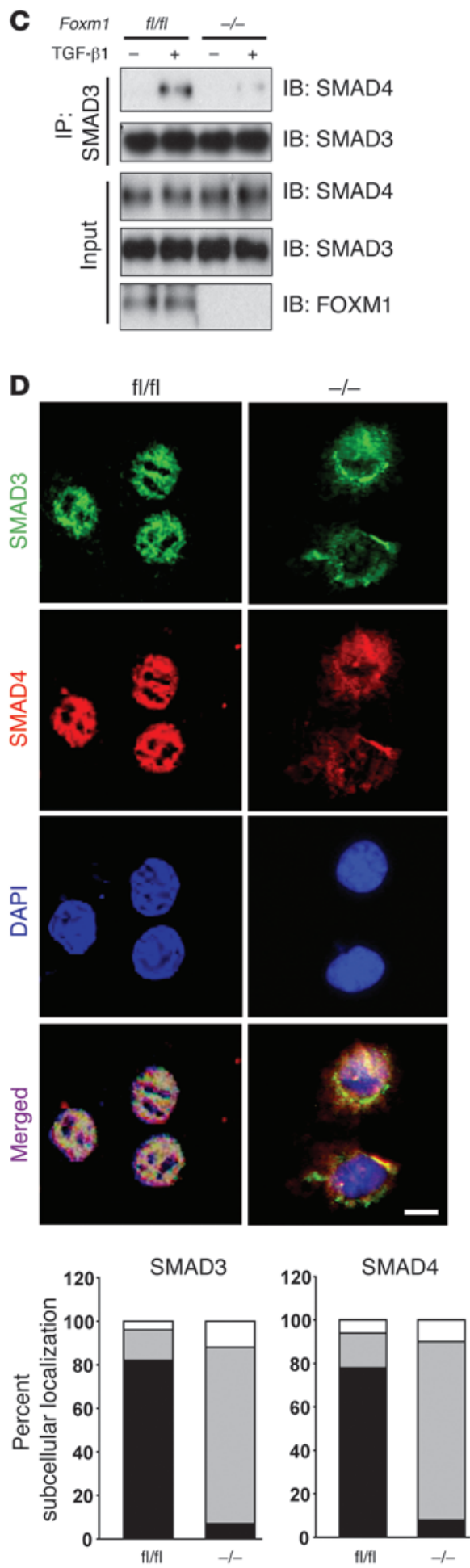

Nuclear

$\square$ Nuclear + cytoplasmic

$\square$ Cytoplasmic
Figure 5

FOXM1 is required for formation of the nuclear SMAD3/SMAD4 complex. (A) FOXM1 knockdown blocked formation of the SMAD3/SMAD4 complex. MDAMB-231 shControl or shFOXM1 cells were treated with TGF- $\beta 1(5 \mathrm{ng} / \mathrm{ml})$ for 1 hour before immunoprecipitation of SMAD3. The amount of SMAD4 bound to SMAD3 was detected by immunoblot using an anti-SMAD4 antibody. (B) MDA-MB-231 shControl or shFOXM1 cells were treated with TGF- $\beta 1$ for 1 hour. The cells were stained with an antiSMAD3 or anti-SMAD4 antibody and then by a rhodamine-conjugated antimouse secondary antibody (red, for SMAD4) or a FITC-conjugated antirabbit secondary antibody (green, for SMAD3), and the nuclei were visualized with DAPI (blue). Scale bar: $20 \mu \mathrm{m}$. Results are indicated at bottom as the percentage of cells showing mostly cytoplasmic or mostly nuclear fluorescence or both. (C) FOXM1 deficiency inhibited formation of the SMAD3/SMAD4 complex. Foxm $1^{\text {tllfl }}$ or Foxm $1^{-/-}$MEFs were treated with TGF- $\beta 1(5 \mathrm{ng} / \mathrm{ml})$ for 1 hour. Then the immunoprecipitation of SMAD3 and the immunoblots of SMAD3 and SMAD4 were conducted as described for panel A. (D) Foxm $1^{1 / / t /}$ or Foxm $1^{-1-}$ MEFs were treated with TGF- $\beta 1$ for 1 hour. Scale bar: $20 \mu \mathrm{m}$. the inhibitory effect of only TIF1 $\gamma$ (Figure 6A and Supplemental Figure 3A). Furthermore, TIF1 $\gamma$ decreased the ability of SMAD4 to interact with SMAD3, whereas FOXM1 overexpression attenuated the inhibitory effect of TIF1 $\gamma$ on SMAD3/SMAD4 complex formation in both cell lines (Figure 6B and Supplemental Figure 3B).
One of the major mechanisms underlying the inhibitory effect of TIF $1 \gamma$ on TGF- $\beta$ signaling is through the acceleration of SMAD 4 monoubiquitination (9). SMAD4 monoubiquitination leads to the dissociation of the SMAD3 and SMAD4 complex and hence the termination of SMADs' complex activity. We found that FOXM1 

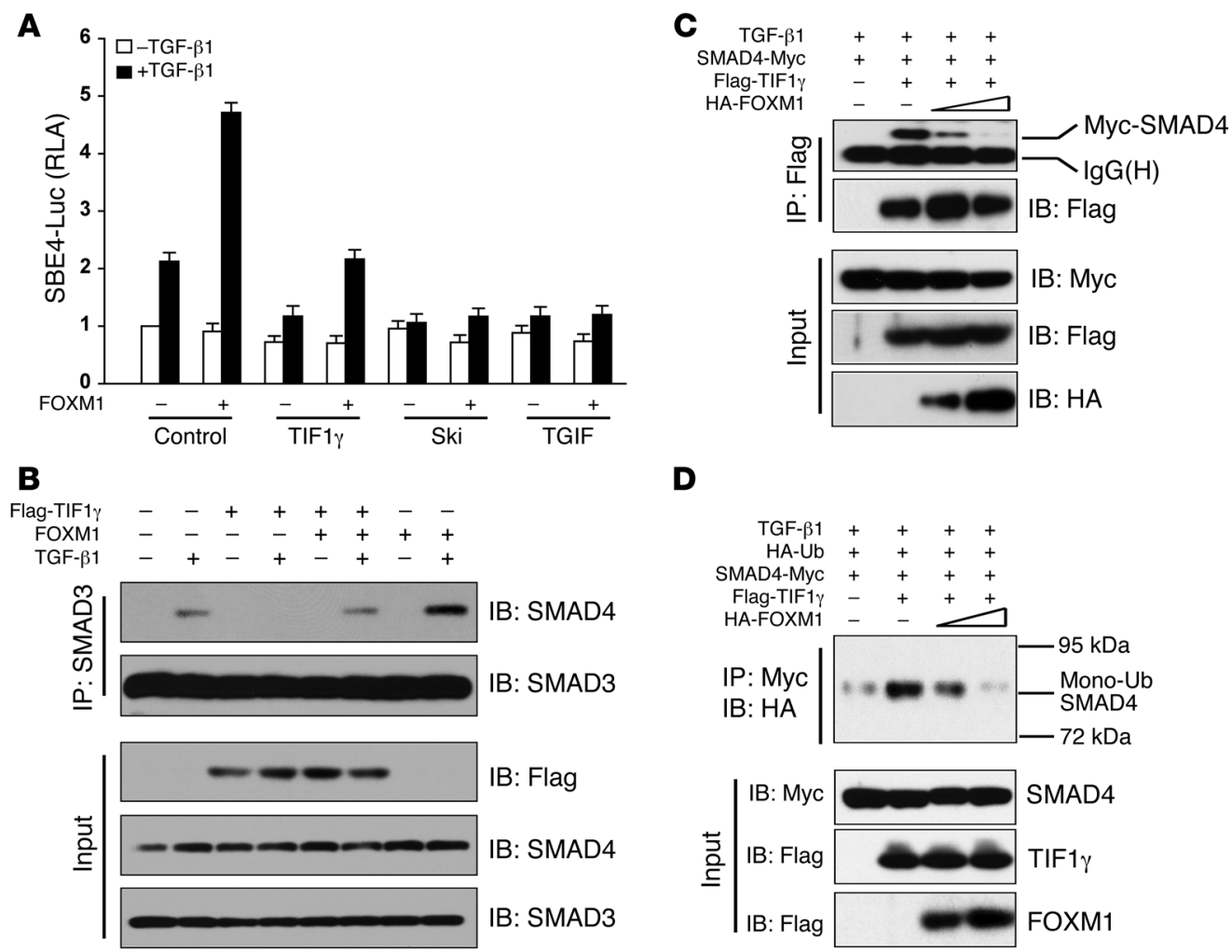

\section{Figure 6}

FOXM1 attenuates the inhibitory effects of TIF1 $\gamma$ on TGF- $\beta$ signaling. (A) FOXM1 attenuated the inhibitory effect of TIF1 $\gamma$ on SMAD3-activated transcription. 4T07 control (-) and FOXM1 overexpression $(+)$ cells were transfected with the indicated plasmids, and then treated with TGF- $\beta 1$ ( $5 \mathrm{ng} / \mathrm{ml}$ ) for 20 hours before being harvested for luciferase assay. (B) FOXM1 relieved the inhibitory effect of TIF1 $\gamma$ on formation of the SMAD3/ SMAD4 complex. 4T07 control (-) and FOXM1 overexpression (+) cells were transfected with plasmids encoding TIF1 $\gamma$ expression constructs. The cells were harvested 48 hours after treatment with TGF- $\beta 1(5 \mathrm{ng} / \mathrm{ml})$ for 2 hours. (C) FOXM1 impaired the exogenous association of SMAD4 with TIF1 $\gamma$. 293T cells were transfected with the indicated plasmids. 48 hours after transfection, cells were harvested for coimmunoprecipitation after incubation with TGF- $\beta 1(5 \mathrm{ng} / \mathrm{ml})$ for 16 hours. (D) FOXM1 inhibited TIF1 $\gamma$-mediated SMAD4 monoubiquitination (Mono-Ub). 293T cells were transfected with the indicated plasmids. Experiments were performed as described for $\mathbf{C}$.

overexpression impaired the TGF- $\beta 1$-induced association of exogenous and endogenous SMAD4 with TIF1 $\gamma$ in $293 \mathrm{~T}$ and breast cancer 4T07 cells, respectively (Figure 6C and Supplemental Figure $3 \mathrm{C})$. The monoubiquitination of SMAD4 was drastically increased by TIF1 $\gamma$; however, this TIF1 $\gamma$ effect was reduced by FOXM1 in both cell lines (Figure 6D and Supplemental Figure 3D).

Next, we found that FOXM1 does not bind to SMAD4 directly but instead formed a complex with SMAD3/SMAD4 (Figure $4 \mathrm{~A}$ ), suggesting that FOXM1 modulates TIF1 $\gamma$ action through SMAD3. Previous studies have shown that TIF1 $\gamma$ can interact with phosphorylated SMAD2/SMAD3 (27) and that TIF1 $\gamma$ monoubiquitinates SMAD4 within the SMAD3/SMAD4 complex with a greater efficiency than free-SMAD4 (8). Thus, we tested whether SMAD3 is critical for the TIF1 $\gamma$ interaction with SMAD4. Indeed, knockdown of SMAD3 significantly decreased the interaction between TIF1 $\gamma$ and SMAD4 in 293T and 4T-07 cells (Figure 7A and Supplemental Figure 3E).

Next, we tested whether FOXM1 competes with TIF1 $\gamma$ to bind to SMAD3. FOXM1 overexpression blocked the endogenous as well as the exogenous TIF $1 \gamma$-SMAD3 interaction (Figure 7B and Supplemental Figure 3, F and G). Moreover, overexpression of the $\mathrm{N}$ terminus of FOXM1, the domain binding to SMAD3, abolished the effects of FOXM1 on the SMAD3/SMAD4 complex formation and on SMAD4 monoubiquitination in BT-474 and 4T07 cells (Figure 7C and Supplemental Figure 3D). This is probably because the $\mathrm{N}$ terminus of FOXM1, which can bind to SMAD3 but cannot prevent TIF1 $\gamma$ from binding to SMAD3 (Supplemental Figure $3 \mathrm{H}$ ), competes with FOXM1 for binding with SMAD3 (Figure 7C). In addition, overexpression of the $\mathrm{N}$-terminal domain of FOXM1 blocked the transcriptional activation of TGF- $\beta 1$-responsive genes mediated by FOXM1 overexpression in BT-474 and 4T07 cells (Supplemental Figure 1D). Together, the above data indicated that SMAD3 modulated the interaction of TIF1 $\gamma$ with SMAD4 and that FOXM1 competitively inhibited the binding of TIF1 $\gamma$ to SMAD3. As a result, FOXM1 interacts with SMAD3 to interfere with the SMAD3/TIF1 $\gamma$ interaction, causing reduced SMAD4/ TIF1 $\gamma$ binding and hence monoubiquitination of SMAD4 to increase the duration of the SMAD3/SMAD4 complex.

TGF- $\beta 1$ induces recruitment of the SMAD3/SMAD4 complex to the SLUG promoter, and knockdown of FOXM1 blocks binding of the SMAD3/ SMAD4 complex to the SLUG promoter. It is well known that SLUG is induced in response to TGF- $\beta 1$ and has an important role in programming cells toward the metastatic phenotype in breast cancer (28-30). Moreover, SLUG has been reported to be required for 
A

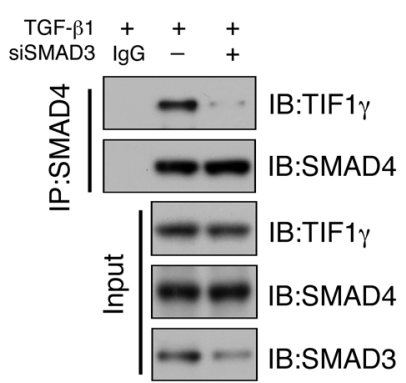

B

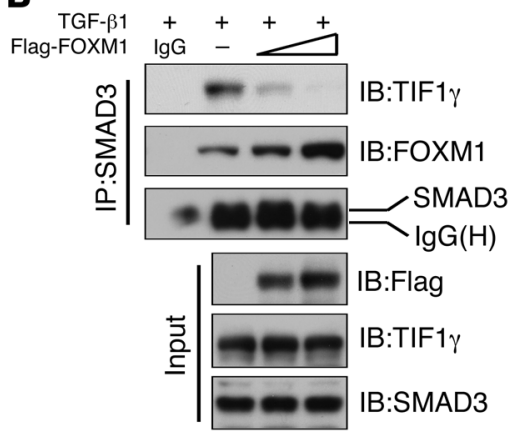

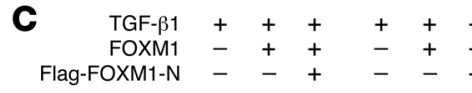

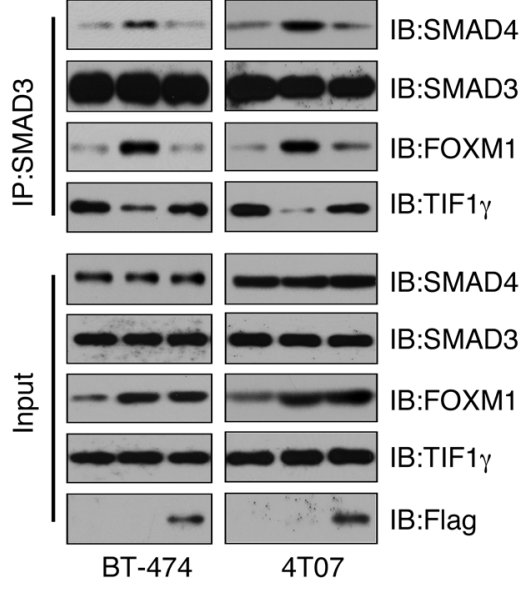

\section{Figure 7}

SMAD3 is critical for the TIF1 $\gamma$ interaction with SMAD4, and FOXM1 competes with TIF1y to bind to SMAD3. (A) Knockdown of SMAD3 blocked the interaction between TIF1 $\gamma$ and SMAD4. 293T cells were transfected with control or SMAD3siRNA. The cells were harvested 48 hours after treatment with TGF- $\beta 1(5 \mathrm{ng} / \mathrm{ml})$ for 2 hours. (B) FOXM1 prevented the association of SMAD3 with TIF1 $\gamma$. 293T cells were transfected with the indicated plasmids. (C) Overexpression of the $\mathrm{N}$ terminus of FOXM1 abolished the effects of FOXM1 on the SMAD3/SMAD4 complex formation. 4T07 or BT-474 control $(-)$ and FOXM1 overexpression $(+)$ cells were transfected with Flag-FOXM1 N-terminal mutant. Cells were harvested 48 hours after 2 hours of treatment with TGF- $\beta 1(5 \mathrm{ng} / \mathrm{ml})$. cell invasion in human breast cancer cells $(29,30)$. Furthermore, we found that the level of SLUG expression is correlated with the level of FOXM1 expression but not with the level of TIF $1 \gamma$ in breast cancer cell lines (Supplemental Figure 1B). Therefore, we hypothesized that FOXM1 regulates the expression of SLUG by regulating SMAD3 activity. We found that the human SLUG promoter contains 5 putative SMAD3-binding elements in 3 regions. Antibody-specific ChIP was used to determine that the region (-416 to $-453 \mathrm{bp}$ ) is able to bind to endogenous SMAD3 protein in MDAMB-231 shControl cells (Figure 8A). However, the other 2 regions ([-136 to $-139 \mathrm{bp}]$ and [ -591 to $-710 \mathrm{bp}]$ ) are incapable of binding to SMAD3 (Supplemental Figure 4A). To determine the specificity of the SMAD3-binding elements, we performed site-specific mutagenesis within the CAGA boxes of region ( -416 to $-453 \mathrm{bp}$ ) of the SLUG promoter luciferase reporter. We then transfected these mutant luciferase reporters into MDA-MB-231 shControl cells and compared their activity with that of the WT SLUG promoter (Supplemental Figure 4B). Disruption of one or both of the CAGA boxes significantly attenuated TGF- $\beta$-induced SLUG promoter activity in the cells (Supplemental Figure 4B), indicating that the response elements in this region are specific for transcriptional activation of SLUG by TGF- $\beta$. Moreover, ChIP and re-ChIP assays showed that TGF- $\beta 1$ induced the binding of SMAD 3 in the SMAD3/SMAD4 complex to this SLUG promoter region in MDAMB-231 shControl cells (Figure 8A), indicating that the SLUG gene is regulated by TGF- $\beta$-mediated activation of SMAD3/SMAD4. In contrast, recruitment of SMAD3 and the SMAD3/SMAD4 complex to the SLUG promoter region was decreased in MDA-MB-231 shFOXM1 cells (Figure 8A). Overexpression of FOXM1 increased the binding ability of SMAD3 and the SMAD3/SMAD4 complex to the SLUG promoter in BT- 474 cells compared with control cells (Supplemental Figure 4C). Further, TGF- $\beta 1$ induced SLUG expression in MDA-MB-231 shControl cells, whereas induction of SLUG expression by TGF- $\beta 1$ was significantly impaired in MDA-MB-231 shFOXM1 cells (Figure 8B). Conversely, overexpression of FOXM1 significantly increased the SLUG expression induced by TGF- $\beta 1$ in BT-474 cells (Supplemental Figure 4D), whereas knocking down of SMAD3 suppressed the effect of FOXM1 in the cells (Supplemental Figure 4D). Knocking down of SMAD3 also abolished the effect of FOXM1 on the SLUG expression induced by TGF- $\beta 1$ in 4T07 cells (Supplemental Figure 4D). Collectively, these results indicate that FOXM1 is important for the maintenance of the SMAD3/SMAD4 complex in the promoter region of TGF- $\beta 1$ target genes and for the induction of target gene expression.

FOXM1 regulates TGF- $\beta$-induced invasion by affecting TIF1 $\gamma$-mediated inbibition of TGF- $\beta$ signaling but not by FOXM1's direct transcriptional property. Next, we examined whether FOXM1 has a role in TGF- $\beta 1$ induced invasion of breast cancer cells because TGF- $\beta / S M A D$ signaling is one of the major pathways that regulate invasion in breast cancer (4). Overexpression of FOXM1 increased the TGF- $\beta 1$ induced invasion of 4T07 and BT-474 cells relative to control cells (Figure 8C). To ascertain that the effect of FOXM1 on the TGF- $\beta 1$ induced invasion is largely dependent on SMAD3, we used breast cancer cells that overexpress FOXM1 but are deficient in SMAD3. Overexpression of FOXM1 promoted the TGF- $\beta 1$-induced invasion in 4T07 cells (Figure 8D). In contrast, knockdown of SMAD3 abolished the effect of FOXM1 overexpression on the TGF- $\beta 1$-induced invasion in 4T07 cells (Figure 8D). These results indicate that FOXM1's effect on TGF- $\beta 1$-induced invasion depends on SMAD3.

Conversely, knockdown of FOXM1 in 4T1 and MDA-MB-231 decreased the TGF- $\beta 1$-induced invasive ability of these cells relative to control cells (Figure 8E). These results indicate that FOXM1 is required for the TGF- $\beta$-induced invasive ability of breast cancer cells. The inhibitory effect of ShFOXM1 on invasion of MDAMB-231 cells was rescued by shRNA-resistant FOXM1 or by shRNAresistant FOXM1 R286A/H287A mutant, which is incapable of DNA binding and thus does not bind to FOXM1 response elements (Figure 8F). This finding indicates that the effect of FOXM1 on TGF- $\beta 1$-induced responses depends on the interaction of FOXM1 with SMAD3 but not on FOXM1's direct transcriptional 

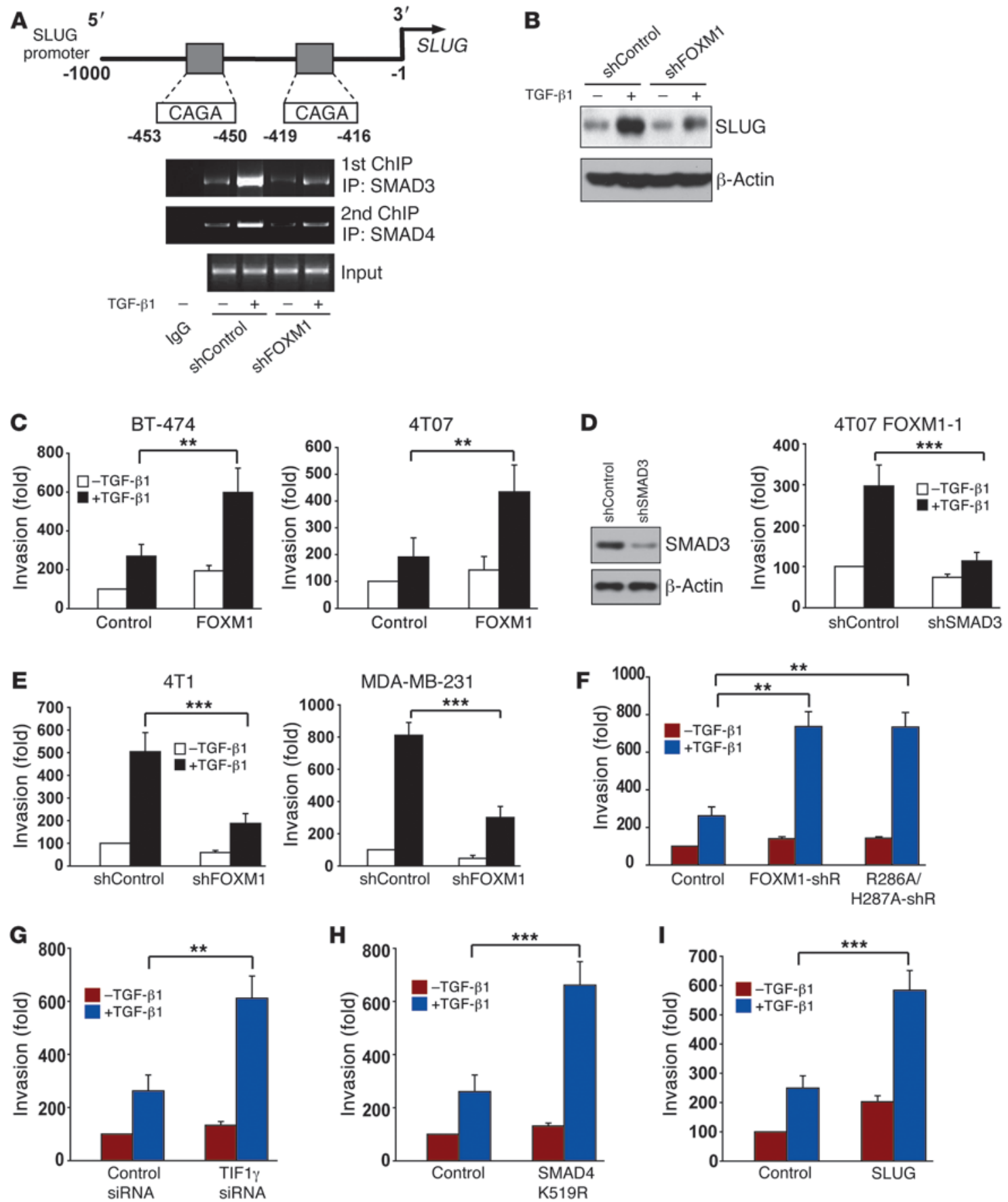

\section{Figure 8}

FOXM1 regulates TGF- $\beta$-induced invasion by affecting TIF1 $\gamma$-mediated inhibition of TGF- $\beta$ signaling but not by FOXM1's direct transcriptional property. (A) Upper panel: diagram showing the putative SMAD3-binding elements in the 1,000-bp genomic DNA sequence of the SLUG promoter. Lower panel: knockdown of FOXM1 blocked the binding of SMAD3 or SMAD3/SMAD4 complex to the SLUG promoter. ChIP and re-ChIP assays were performed in MDA-MB-231 shControl or shFOXM1 cells that were incubated with or without TGF- $\beta 1$ (5 ng/ml) for 24 hours. (B) Knockdown of FOXM1 inhibited expression of SLUG induced by TGF- $\beta 1$ in MDA-MB-231 shControl or shFOXM1 cells. (C) 4 T07 and BT-474 control and FOXM1 overexpression cells were subjected to in vitro invasion assay with or without TGF- $\beta 1$ ( 5 ng/ml) for 16 hours. Each column indicates the mean $( \pm \mathrm{SD})$ results of 2 independent experiments. ${ }^{* \star} P<0.01$. (D) Left, 4T07-FOXM1-1 cells were transduced with shControl or shSMAD3 viruses. Right, 4T07-FOXM1-1 shControl and shSMAD3 cells were subjected to in vitro invasion assay with or without TGF- $\beta 1$. ${ }^{* * *} P<0.001$. (E) 4T1 and MDA-MB-231 shControl and shFOXM1 cells were subjected to in vitro invasion assay with or without TGF- $\beta 1$ (5 ng/ml). ${ }^{* * *} P<0.001$. (F) MDA-MB-231 shFOXM1 cells were transduced with control or shRNA-resistant FOXM1 (FOXM1-shR) or its mutant shRNAresistant R286A/H287A (R286A/H287A-shR) viruses. ${ }^{*} P<0.01$. (G) MDA-MB-231 shFOXM1 cells were transfected with siControl or siTIF1 $\gamma$. ${ }^{* *} P<0.01$. (H) MDA-MB-231 shFOXM1 cells were transfected with control or the SMAD4 K519R mutant. ${ }^{* *} P<0.001$. (I) MDA-MB-231 shFOXM1 cells were transfected with control or the SLUG expression vector. ${ }^{* * *} P<0.001$. 


$\begin{aligned} & \text { Table } 1 \\ & \text { Knockdown of FOXM1 inhibits lung metastasis formation in vivo }\end{aligned}$
$\begin{array}{lcc} & & \\ \text { Cell line } & \text { Incidence } & \text { Mean (range) } \\ \text { 4T1-WT } & 5 / 5 & 71.6(18-135) \\ \text { 4T1-shControl } & 5 / 5 & 94.6(16-168) \\ \text { 4T1-shFOXM1-1 } & 1 / 5 & 0.8(0-4)^{\mathrm{A}} \\ \text { 4T1-shFOXM1-2 } & 3 / 5 & 16.2(0-63)^{\mathrm{B}} \\ \text { MDA-MB-231-WT } & 5 / 5 & 83.0(47-150) \\ \text { MDA-MB-231-shControl } & 5 / 5 & 62.0(17-101) \\ \text { MDA-MB-231-shFOXM1-1 } & 3 / 5 & 2.4(0-40)^{\mathrm{A}} \\ \text { MDA-MB-231-shFOXM1-2 } & 3 / 5 & 3.8(0-10)^{\mathrm{A}}\end{array}$

Results are expressed as mean number and range of lung metastatic nodules. Incidence indicates the number of mice with lung metastases divided by the total number of mice injected with tumor cells. Results shown are for 1 representative experiment of 2 . ${ }^{A} P<0.01$; ${ }^{B} P<0.05$ vs. WT groups.

property. On the other hand, depletion of FOXM1 in MDAMB-231 cells substantially decreased TGF- $\beta 1$-induced invasion (Figure 8E). The inhibitory effect of shFOXM1 on MDA-MB-231 cell invasion was rescued by knockdown of TIF1 $\gamma$ (Figure 8G) and by the SMAD4 K519R mutant (Figure 8H). Mutation of $\mathrm{K} 519 \mathrm{R}$ in SMAD4 resulted in a nonubiquitinated form of SMAD4, which cannot be monoubiquitinated by TIF1 $\gamma(9)$, and hence its activation is no longer dependent on FOXM1, indicating that FOXM1 facilitates TGF- $\beta 1$-induced invasion by attenuating the inhibitory effect of TIF $1 \gamma$.

We then investigated whether SMAD4 is required for invasion of breast cancer cells, since FOXM1 affects TIF1 $\gamma$-mediated SMAD4 monoubiquitination. Depletion of SMAD4 in MDA-MB-231 and 4T1 cells, which express high levels of FOXM1, substantially decreased the TGF- $\beta 1$-induced invasion (Supplemental Figure 4, $\mathrm{E}$ and $\mathrm{F})$. To further test that the effect of FOXM1 on the TGF- $\beta 1-$ induced invasion depends on SMAD4, we used breast cancer cells that overexpress FOXM1 but are deficient in SMAD4. Overexpression of FOXM1 promoted the TGF- $\beta 1$-induced invasion in $4 \mathrm{~T} 07$ and BT-474 cells, whereas knockdown of SMAD4 abolished the effect of FOXM1 overexpression on the TGF- $\beta 1$-induced invasion (Supplemental Figure 4, G and $\mathrm{H}$ ). These results indicate that FOXM1's effect on TGF- $\beta 1$-induced invasion depends on SMAD4.

We found that the inhibitory effect of shFOXM1 on invasion was rescued by a SLUG expression vector (Figure 8I), supporting the notion that SLUG is a downstream effector of FOXM1 on cell invasion. These findings were also observed in 4T1 cells (Supplemental Figure 4, I-K), suggesting that the above findings are not limited to MDA-MD-231 cells. Collectively, these data indicate that FOXM1 plays a crucial role in the regulation of TGF- $\beta 1$-induced invasion of breast cancer cells through the TGF- $\beta 1$-SMAD3/SMAD4-SLUG axis.

FOXM1/SMAD3 pathway promotes breast cancer metastasis. Knockdown of FOXM1 in 4T1 cells significantly reduced their metastatic ability; the parental or shControl cells formed lung metastases in $100 \%$ of the mice, with averages of 71 and 94 visible metastases per mouse, respectively (Table 1), whereas shFOXM1-1 and shFOXM1-2 cells produced lung metastases in $20 \%$ and $60 \%$ of mice, respectively, with averages of 0.8 and 16 visible metastases per mouse, respectively (Table 1 ). Similarly, the ability of MDA-MB-231 cells to metastasize to the lung in nude mice was substantially inhibited by knockdown of FOXM1 (Table 1). Thus, inhibition of FOXM1 suppressed metastasis formation by otherwise highly malignant cancer cells. Moreover, we analyzed the metastatic samples from the above animal experiments using immunostaining for nuclear SMAD3 level with an antibody specific for phosphorylated SMAD3. The nuclear SMAD3 level was decreased in lung metastatic lesions formed by shFOXM1 cells relative to that seen in lung metastatic lesions formed by $4 \mathrm{~T} 1$ and MDA-MB-231 cells (Supplemental Figure 5, A and B), suggesting that the level of nuclear SMAD3 is regulated by FOXM1 and is related to the metastatic phenotype. Furthermore, we found that the inhibitory effect of shFOXM1 on metastatic ability of 4T1 and MDA-MB-231 cells was rescued by shRNA-resistant FOXM1 R286A/H287A, which is incapable of DNA binding, indicating that the effect of FOXM1 on metastasis does not depend on FOXM1's direct transcriptional property in vivo (Table 2).

Conversely, 4T07 parental cells did not produce spontaneous lung metastases when implanted into the mammary fat pads of mice (Table 3 ). In contrast, FOXM1-transfected 4T07 cells (FOXM1-1 and FOXM1-2) produced lung metastases in 70\% to $80 \%$ of the mice (Table 3 ). Furthermore, the level of nuclear SMAD3 was highly elevated in lung metastatic lesions formed by FOXM1-transfected 4T07 cells relative to the level in lung tissue from the mice injected with 4T07 control cells (Supplemental Figure 5C). Examination of the mouse livers revealed that FOXM1 also increased formation of liver metastases (Supplemental Figure 5D). Thus, overexpression of FOXM1 mediated metastasis formation by otherwise nonmetastatic cancer cells. These data indicated that FOXM1 is responsible for the metastasis formation and for the increase in nuclear SMAD3 seen in 4T07 cells.

To further ascertain whether the metastasis-promotion effect of FOXM1 is largely dependent on SMAD3, we used breast cancer cells that overexpress FOXM1 but are deficient in SMAD3. Overexpression of FOXM1 in 4T07 cells promoted lung metastases (Table 4). However, SMAD3 knockdown in 4T07-FOXM1 cells diminished their metastatic ability (Table 4), indicating that metastatic promotion by FOXM1 depends on SMAD3 in vivo. Together, the above results demonstrate that FOXM1 signals through SMAD3 to promote the metastasis of breast cancer cells.

Expression of FOXM1 is correlated with p-SMAD 3 and SLUG expression and associated with metastasis in human breast cancers. We analyzed the clinical relevance of FOXM1 in tumor progression and metastasis by examining the expression levels of FOXM1, p-SMAD3, SMAD4, TIF1 $\gamma$, and SLUG in 12 samples of normal breast tissue and in 36 paired samples of breast primary tumor and matched lymph node metastases by immunohistochemical staining. Interestingly, the

\section{Table 2}

The shRNA-resistant R286A/H287A F0XM1 mutant rescues the inhibitory effect of shFOXM1 on metastases in vivo

$\begin{array}{lcc}\text { Cell line } & \text { Incidence } & \text { Mean (range) } \\ \text { 4T1-shFOXM1-1+Control vector } & 2 / 10 & 1.6(0-12) \\ \text { 4T1-shFOXM1-1+R286A/H287A-shR } & 8 / 9 & 62.8(0-108)^{\mathrm{A}} \\ \text { MDA-MB-231-shFOXM1-1+Control vector } & 4 / 9 & 3.6(0-36) \\ \text { MDA-MB-231-shFOXM1-1+R286A/H287A-shR } & 9 / 10 & 56.0(0-112)^{\mathrm{A}}\end{array}$

The lung metastases were analyzed. ${ }^{\mathrm{A}} P<0.01 \mathrm{vs}$. control groups. 
Table 3

FOXM1 is responsible for lung metastasis formation in vivo

$\begin{array}{lcc}\text { Cell line } & \text { Incidence } & \text { Mean (range) } \\ \text { 4T07-WT } & 0 / 10 & 0(0) \\ \text { 4T07-Control } & 0 / 10 & 0(0) \\ \text { 4T07-FOXM1-1 } & 8 / 10 & 27.8(0-56)^{\mathrm{A}} \\ \text { 4T07-FOXM1-2 } & 7 / 10 & 43.2(0-73)^{\mathrm{B}}\end{array}$

The lung metastases were analyzed. ${ }^{A} P<0.05 ;{ }^{B} P<0.01$ vs. WT groups.

level of TIF1 $\gamma$ expression was not associated with metastatic status (Figure 9, A and B), although TIF1 $\gamma$ expression was slightly lower in tumor tissues than in normal breast tissues (Supplemental Figure 6A). In contrast, FOXM1 expression was significantly associated with metastasis (Figure 9, A and B), indicating that the level of FOXM1, but not the level of TIF1 $\gamma$, is important for metastasis. As for p-SMAD3 and SMAD4, 44\% of the breast primary tumors expressed p-SMAD3, but $86 \%$ of the metastases expressed p-SMAD3, and $89 \%$ of the breast primary tumors expressed SMAD 4 , but $94 \%$ of the metastases expressed SMAD4. Moreover, the expression levels of p-SMAD3 and nuclear SMAD4 were higher in the metastases than in primary tumors (Supplemental Figure 6B). Notably, expression of FOXM1, p-SMAD3, and the p-SMAD 3 target gene SLUG was correlated to a significantly greater degree with breast cancer metastasis than with primary breast cancer (Figure 9, A and B). Furthermore, the expression levels of FOXM1 directly correlated with those of p-SMAD3 and SLUG (Table 5). Together, our data underscore the clinical relevance of FOXM1 in regulating activation of TGF- $\beta$ signaling in breast cancer metastasis.

\section{Discussion}

In this report, we demonstrate that the FOXM1-SMAD3 interaction represents a critical mechanism for controlling TGF- $\beta$ signaling and breast cancer cell invasion and metastasis. FOXM1 sustains TGF- $\beta$-induced formation of the SMAD3/SMAD4 complex and enhances SMAD3-mediated transcriptional activity in breast cancer cells. TGF- $\beta$ signaling is initiated by the induction of SMAD3 phosphorylation, and phosphorylated SMAD3 forms a complex with SMAD4 that is then translocated to nucleus. For the termination of TGF- $\beta$ signaling, as a feedback mechanism, TGF- $\beta /$ SMAD 3 induces the binding of TIF $1 \gamma$ to SMAD4, causing the monoubiquitination of SMAD4 and hampering the ability of SMAD4 to form a stable complex with activated SMAD3. In the presence of FOXM1, SMAD3 interacts with FOXM1. This prevents the interaction between TIF $1 \gamma$ and SMAD3 and thus the monoubiquitination of SMAD 4 by TIF $1 \gamma$, thereby sustaining the complex of SMAD3/SMAD4 in the nucleus. Retention of SMAD3 in the nucleus leads to increased TGF- $\beta /$ SMAD3-mediated transcriptional activity, target gene expression, and breast cancer metastasis (Figure 9C). Conversely, knockdown of FOXM1 in breast cancer cells impairs TGF- $\beta$-induced SMAD3/SMAD4 complex formation and target gene expression, resulting in defects in cell invasion and metastasis (Figure 9C). Genetic deletion of FOXM1 in MEF cells also abolishes TGF- $\beta$-induced SMAD3/SMAD4 complex formation and transcriptional activity. This finding is underscored by the important clinical observation that FOXM1 expression was positively correlated with the level of p-SMAD3 and its downstream target SLUG in metastatic breast cancer, which may indi- cate the relevant molecular mechanism by which the p-SMAD3 signal is sustained during cancer progression and metastasis.

Tumor cells usually secrete abundant TGF- $\beta$, which promotes invasion and metastasis (31). FOXM1 is also frequently aberrantly activated in many human cancers, including breast cancer $(17,32)$. Specific and diverse TGF- $\beta$ functions depend on the SMADs as a central node for the integration of TGF- $\beta$ signaling with other signaling pathways $(1,33)$. In the present study, we found that FOXM1 specifically interacts with SMAD3, which supports the recent observations that SMAD3, but not SMAD2, is required for TGF- $\beta$ induced metastasis in breast cancer (34). Our study demonstrates that FOXM1 enhances the nuclear retention of SMAD3 by maintaining the SMAD3/SMAD4 complex. Considering that FOXM1 primarily binds with SMAD3 in the nucleus, combined with our finding that PPM1A-dephosphorylated SMAD3 is not affected by FOXM1, we reasoned that the role of FOXM1 in TGF- $\beta$ signaling is to act after SMAD3 activation and before SMAD3 inactivation by dephosphorylation. These findings are consistent with the observation of a strong correlation between FOXM1 and p-SMAD3 levels in human breast cancer tissues because p-SMAD3 is mainly located in the nucleus. More importantly, they also provide a mechanism for this observation in human breast cancer tissues. However, it was reported that the expression of the TGF- $\beta$ type II receptor in lung vasculature in FOXM1 gene deletion embryos is reduced (35). Future studies along this line will determine whether this is either a cell type-specific or tumor-specific regulation or both.

TIF1 $\gamma$ has been shown to be a tumor suppressor in the pancreas. TIF1 $\gamma$ expression was markedly downregulated in human pancreatic tumors, and inactivation of TIF $1 \gamma$ was involved in the formation of cystic tumors of the pancreas (36). Whether the TIF1 $\gamma$ gene is downregulated in human breast cancers is unknown. Our results indicate that although TIF1 $\gamma$ expression was slightly lower in breast cancer tissues than in normal breast tissues, the level of TIF1 $\gamma$ expression in the tissues was not associated with metastasis. In contrast, we found that FOXM1 expression was significantly associated with metastasis. Unexpectedly, we found that FOXM1 promoted formation of the SMAD3/SMAD 4 complex by antagonizing TIF1 $\gamma$ activity. This result is consistent with reports that TIF1 $\gamma$ regulates TGF- $\beta$ signaling by disrupting formation of the SMAD3/SMAD4 complex $(8,9,27)$. Moreover, several recent studies clearly show that as a SMAD 4 monoubiquitin ligase, TIF1 $\gamma$ inhibits TGF- $\beta$ signaling $(8,9)$. Thus, we focused on whether FOXM1 affects the monoubiquitination of SMAD 4 induced by TIF $1 \gamma$. Indeed, the TIF $1 \gamma$-induced monoubiquitination of SMAD4 was decreased by FOXM1 expression in a dose-dependent manner. However, it has been shown that TIF1 $\gamma$ competes with SMAD4 for binding to SMAD2 and SMAD3 and forms TIF1 $\gamma /$ SMAD2/SMAD3 complexes regulating SMAD4independent TGF- $\beta$ responses $(27,37)$. TIF1 $\gamma$ loss of function inhibited pancreatic tumorigenesis in the absence of SMAD4 (38).

\section{Table 4}

SMAD3 knockdown in 4T07-FOXM1 cells diminishes the FOXM1-induced metastasis in vivo

$\begin{array}{lcc}\text { Cell line } & \text { Incidence } & \text { Mean (range) } \\ \text { 4T07-FOXM1-1+shControl } & 8 / 10 & 32.6(0-63) \\ \text { 4T07-FOXM1-1+shSMAD3 } & 1 / 10 & 5.8(0-20)^{\mathrm{A}}\end{array}$

The lung metastases were analyzed. ${ }^{A} P<0.01$ vs. shControl groups. 
A
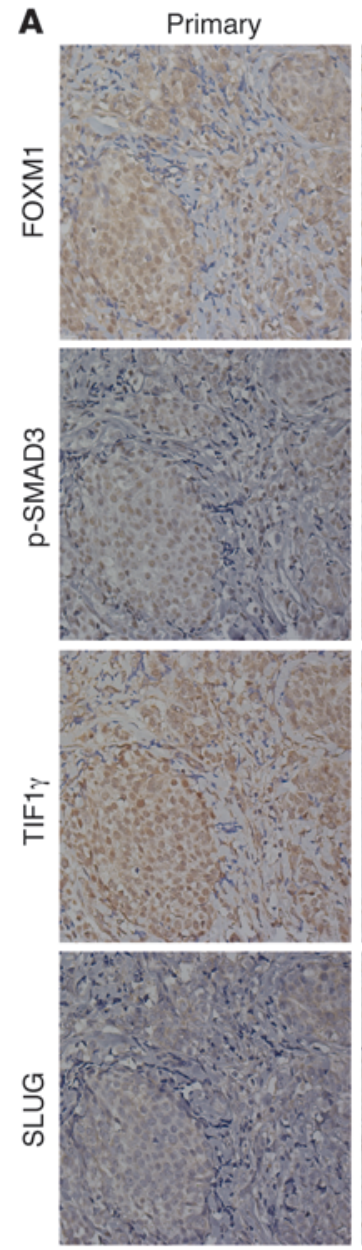

C

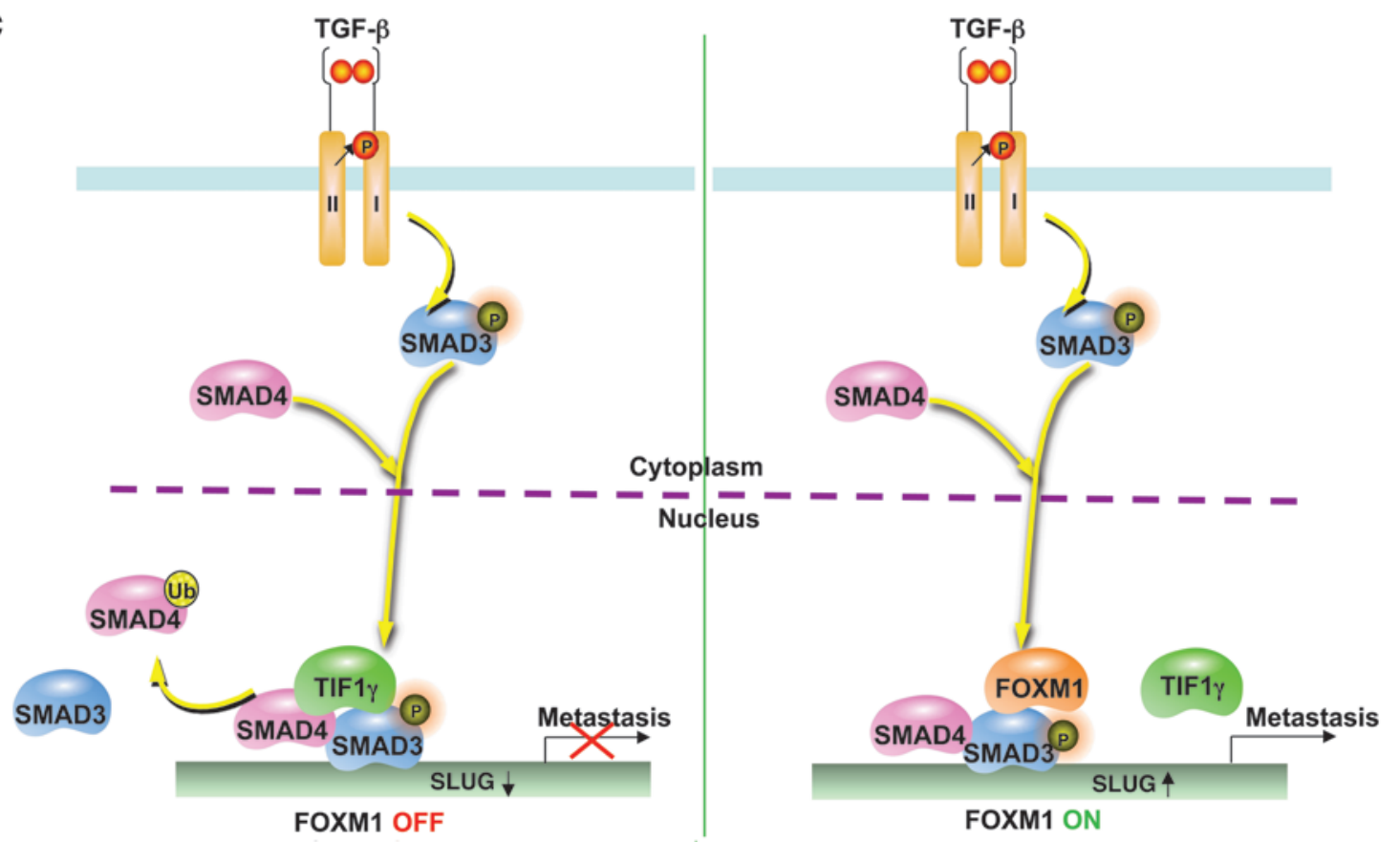

Metastases
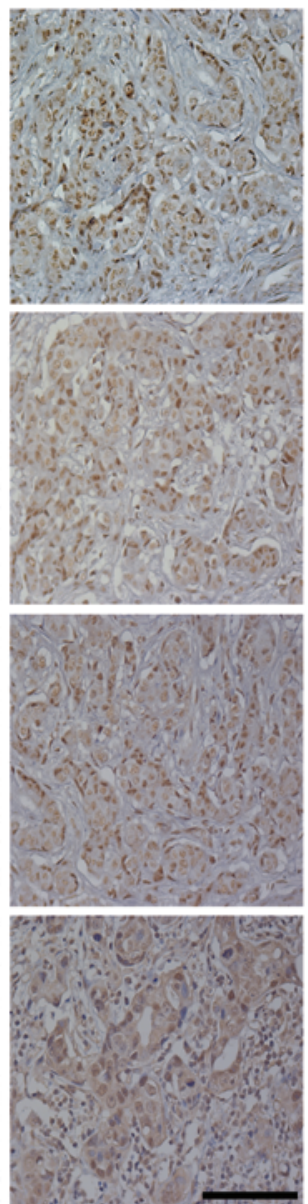
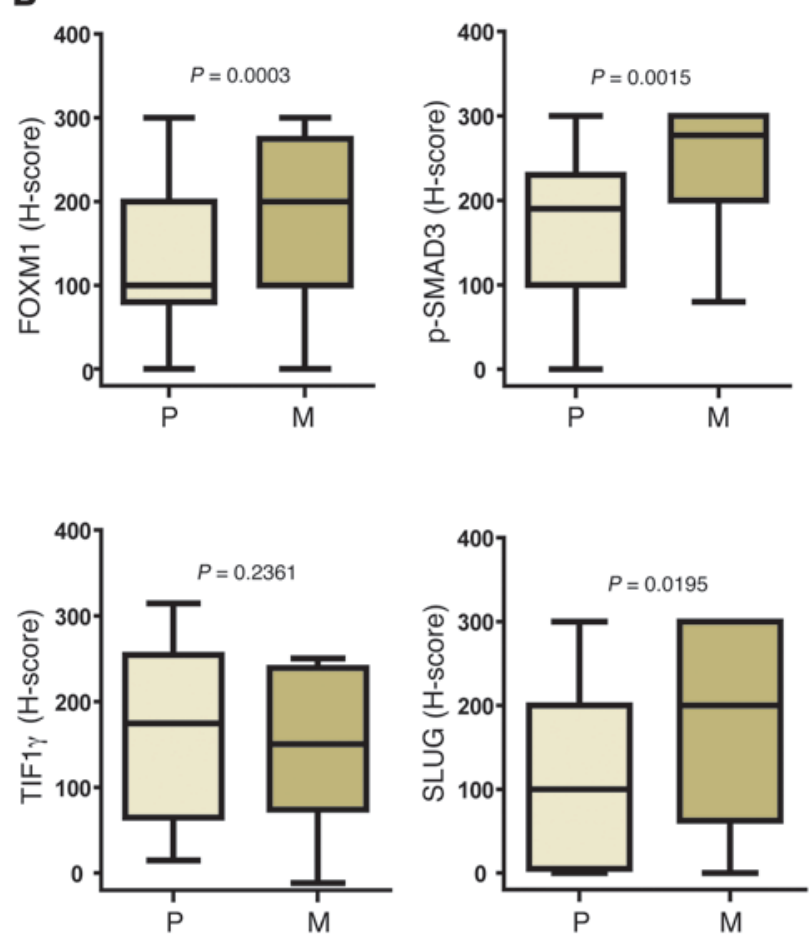


\section{Figure 9}

FOXM1 expression significantly correlates with activated TGF- $\beta$ signaling in human breast cancer. (A) The expression of FOXM1, p-SMAD3, TIF1 $\gamma$, and SLUG in representative cases of primary breast tumors (P) and matched lymph node metastasis tissue specimens (M). Original magnification, $\times 400$. Scale bars: $200 \mu \mathrm{m}$. (B) Boxes indicate interquartile range. Bars from each box extend to the largest and smallest observations. (C) In the working model of interaction of FOXM1 with SMAD3 and SMAD4, FOXM1 promotes metastasis via the TGF- $\beta 1$ pathway. Left: in the absence of FOXM1, TIF1 $\gamma$ sets up a negative-feedback mechanism to the TGF- $\beta 1$ pathway by inducing monoubiquitination of SMAD4 and thus disrupting formation of the SMAD3/SMAD4 complex. Thus, SMAD3 is not retained in the nucleus, which decreases SLUG expression and prevents metastasis. Right: in the presence of FOXM1, FOXM1 interacts with SMAD3/SMAD4, which results in inhibition of binding of TIF1 $\gamma$ with SMAD4 and thus prevention of monoubiquitination of SMAD4 by TIF1 $\gamma$. Thus, p-SMAD3 is retained in the nucleus via sustenance of the SMAD3/SMAD4 complex, which ultimately promotes activation of SLUG transcription and metastasis.

Our study indicated that FOXM1 could compete with SMAD3 for binding to TIF1 $\gamma$. It is of interest to investigate whether FOXM1 also regulates SMAD4-independent TGF- $\beta$ responses during tumor progression and whether it is organ specific.

It has been reported that FOXM1 enhances the metastatic potential of hepatocellular carcinomas. FOXM1b activates the Akt/ Snail1 pathway and stimulates expression of stathmin, lysyl oxidase, and other genes involved in metastasis of hepatocellular carcinoma (16). However, prior to this report, it was unknown whether FOXM1 promotes metastasis of breast cancer. Here, we have demonstrated that FOXM1 promotes metastasis of breast cancer by positively regulating TGF- $\beta$ signaling. Because some reports have indicated that SLUG, but not Snail, seems to be required for invasion of human breast cancer cells $(29,30)$, in this study, we focused on whether FOXM1 regulates the expression of SLUG. We found that the SMAD3/SMAD4 complex was recruited to the SLUG promoter upon treatment with TGF- $\beta$ and that this recruitment was decreased in FOXM1 knockdown cells. Deficiency of FOXM1 impaired the recruitment of the SMAD3/SMAD4 complex to the SLUG promoter, the expression of SLUG, and cell invasiveness, and SLUG restoration rescued the defect in cell invasiveness. Further, a recent study showed that siRNA-mediated inhibition of FOXM1 prevented TGF- $\beta$-induced EMT in lung epithelial adenocarcinoma A549 cells in vitro (39). It is of interest to investigate whether the mechanism by which FOXM1 sustains the TGF- $\beta$-induced formation of the SMAD3/SMAD4 complex underlies the observation.

In summary, our findings indicate that FOXM1 interacts with the transcription factor SMAD3 and promotes its functions and therefore is critical for TGF- $\beta$-mediated gene expression, cell invasion, and cancer metastasis. In recent years, many studies have demonstrated the therapeutic antimetastatic potential of various TGF- $\beta$ antagonists (40). Our findings that overexpression of FOXM1 promotes cancer cell invasion and metastasis, whereas its deficiency restricts cancer metastasis, provide insights into mechanisms and strategies for therapeutic intervention of the TGF- $\beta$ pathway and cancer metastasis.

\section{Methods}

Cell culture and transfection. HEK 293T, MDA-MB-231, BT-474 cells, and immortalized FOXM1 conditional knockout Foxm $1^{f / / f l}$ MEFs were grown in DMEM containing 10\% FBS (HyClone). HaCaT cells were grown in
MEM supplemented with 10\% FBS. 4T07 and 4T1 cells were maintained in DMEM/Ham's F-12 medium supplemented with $10 \%$ FBS. Cells were either transfected with FuGENE HD (Roche) or using X-tremeGENE HP (Roche). For siRNA interference, cells were transfected using Lipofectamine RNAiMAX (Invitrogen). For more information, see Supplemental Experimental Procedures.

Immunoprecipitation and immunoblotting analysis. For coimmunoprecipitation, cells were grown in 10-cm dishes and transfected with the appropriate plasmids. Cell lysates were incubated with $2 \mu \mathrm{g}$ of antibody on a rotator overnight at $4^{\circ} \mathrm{C}$. The protein-antibody-protein $\mathrm{A} / \mathrm{G}$ agarose complexes were prepared by adding $50 \mu \mathrm{l}$ of protein $\mathrm{A} / \mathrm{G}$-agarose beads (Amersham Biosciences) for 1 hour at $4^{\circ} \mathrm{C}$. After extensive washing with RIPA lysis buffer, the immunoprecipitated complexes were resuspended in reducing sample buffer and boiled for 10 minutes. After centrifugation to pellet the agarose beads, supernatants were subjected to SDS-PAGE and immunoblotting, as previously described (20). For more information, see Supplemental Experimental Procedures.

Promoter reporters and dual-luciferase assay. For TGF- $\beta 1$-induced transcriptional reporter assays, HaCaT cells, MDA-MB-231 cells, or Foxm $1^{f^{l / f l} \mathrm{MEFs}}$ were transfected with one of the SBE4-Luc, or ARE-Luc reporter plasmids. Transfection efficiency was normalized by cotransfection with the pRL-TK plasmid. Luciferase activity was measured using a Dual Luciferase Assay System (Promega) as described previously (20).

Quantitative real-time RT-PCR. Quantitative real-time RT-PCR analysis of the SMAD7 and PAI-1 genes was performed using total RNA and the SYBR green reagent with an ABI Prism 7000HT sequence detection system. The sequences of the PCR primers were as follows: SMAD7 forward, $5^{\prime}$-CCCCATCACCTTAGCCGACTCTGC-3'; SMAD7 reverse, 5'-CCCAGGGGCCAGATAATTCGTTCC-3'; PAI-1 forward, 5'-ATTCAAGCAGCTATGGGATTCAA-3'; PAI-1 reverse, 5'-CTGGACGAAGATCGCGTCTG-3'; GAPDH forward, 5'-AATCCCATCACCATCTTCCA-3'; and GAPDH reverse, 5'-TGGACTCCACGACGTACTCA- 3 '. The results were determined using the Comparative $\mathrm{C}_{\mathrm{t}}$ method with the housekeeping gene GAPDH as a control.

Immunofluorescence analysis. Cells were rinsed twice with PBS, fixed with $4 \%$ buffered paraformaldehyde, and permeabilized with $0.5 \%$ Triton X-100 for 15 minutes. Cells were then incubated with the primary antibody overnight at $4{ }^{\circ} \mathrm{C}$ and then with the secondary antibody conjugated to rhodamine or FITC (Molecular Probes) for 1 hour at room temperature. Cells were examined using a deconvolutional microscope (Zeiss).

Heterokaryon assay. HaCaT stable cells expressing FOXM1 were transfected with Flag-SMAD3 plasmids. 24 hours later, the cells were replated together with NIH 3T3 cells. After coculture overnight, $100 \mu \mathrm{g} / \mathrm{ml}$ cycloheximide (Sigma-Aldrich) was added to the culture medium to block protein synthesis. Then the cells were treated with TGF- $\beta 1(5 \mathrm{ng} / \mathrm{ml})$ for 1 hour. Cell fusion was induced by 50\% PEG 1500 (Roche) treatment for 2 minutes. PEG solution was then removed completely, and cells were washed 3 times with PBS followed by an additional 2 hours culture in the presence of cycloheximide. Cells were then fixed and processed for immunofluorescence analysis.

\section{Table 5}

The expression levels of FOXM1 correlate with those of $p$-SMAD3 and SLUG

\begin{tabular}{lclll} 
Correlation & \multicolumn{2}{c}{ p-SMAD3 } & \multicolumn{2}{c}{ SLUG } \\
F0XM1 & $r=0.722$ & $P<0.001$ & $r=0.618$ & $P<0.001$ \\
p-SMAD3 & & & $r=0.653$ & $P<0.001$
\end{tabular}

The correlations were significant as determined by Pearson correlation. 
In vivo ubiquitination assay. $293 \mathrm{~T}$ cells were transfected with HA-ubiquitin constructs together with the indicated plasmids. Forty-eight hours after transfection, cells were harvested by sonication in ubiquitination-lysis buffer (50 mM HEPES [pH 7.8], 200 mM NaCl, 5 mM EDTA, 1\% NP40, 5\% glycerol, $1 \mathrm{mM}$ dithiothreitol, protease inhibitor cocktail [Sigma-Aldrich] and $250 \mathrm{ng} / \mathrm{ml}$ ubiquitin-aldehyde [CalBiochem]).

ChIP and re-ChIP assays. MDA-MB-231 or BT- 474 cells $\left(4 \times 10^{7}\right)$ were prepared with the ChIP assay kit (Cell Signaling Technology). The resulting precipitated DNA samples were analyzed by PCR to amplify a 200-bp region of the SLUG promoter with the following primer pairs: 5 '-TGCCACTTCCAAATATAGGCTCTCA-3' (sense) and 5'-CTGGGACAGCTGTGAACAGA-3' (antisense). In the re-ChIP assay, the DNA complexes were first immunoprecipitated using SMAD3 antibody and then eluted with re-ChIP buffer and immunoprecipitated again using SMAD4 antibody.

In vitro Transwell invasion assay. Invasion assay was carried out in Transwell chambers containing polycarbonate filters $(8-\mu \mathrm{m}$ pore size; BD Biosciences), in which the upper surfaces of the filters in invasion chambers were coated with a growth factor-reduced Matrigel matrix. Breast cancer cells $\left(3 \times 10^{4}\right)$ in a $500 \mu \mathrm{l}$ volume of serum-free medium were placed in the upper chambers and incubated at $37^{\circ} \mathrm{C}$ for 16 hours for invasion assay. The cells that penetrated through Matrigel-coated filters were counted at a magnification of $\times 200$ in 15 randomly selected fields, and the mean number of cells per field was recorded. Each assay was performed on duplicate filters, and the experiments were repeated twice.

Human tissue samples and immunohistochemical analysis. Tissue arrays of normal human breast tissues, human breast primary carcinomas, and matched metastases in lymph nodes (BRM961; US Biomax Inc.) were used in this study. Immunohistochemical analyses were performed on the tissue arrays by a standard immunostaining protocol as previously described (20). Staining was scored by 2 investigators blinded to the clinical data, using a 4-tiered system that incorporated the intensity of immunostaining and the percentage of cells positive. The intensity of immunostaining was scored as follows: negative, 0 ; weak, $1+$; moderate, 2+; and intense, $3+$. For tumors that showed heterogeneous staining, the predominant pattern was taken into account for scoring. The percentage of positive tumor cells and the staining intensity were multiplied to produce a weighted score for each case. The theoretical limits of the scores ranged from 0 ( $0 \%$ of cells staining) to 300 (100\% of the cells staining at $3+$ intensity).

In vivo model of metastases. Pathogen-free female $\mathrm{BALB} / \mathrm{c}$ or nude mice were purchased from the National Cancer Institute. MDA-MB-231 cells $\left(1 \times 10^{6} \mathrm{cells} /\right.$ mouse $)$ were injected into the lateral tail vein of mice. 4T07 $\left(1 \times 10^{5}\right.$ cells/mouse $)$ or $4 \mathrm{~T} 1\left(5 \times 10^{3}\right.$ cells $/$ mouse $)$ cells were injected into the mammary fat pad of mice. The mice were killed when they were moribund or on day 64 after tail vein injection or on day 25 after mammary fat pad injection. The number of tumor nodules on the surface of each lung or liver was counted using a dissecting microscope.

Statistics. The significance of the data from patient specimens was determined by the $\chi^{2}$ test or the Pearson correlation coefficient test. The significance of the in vitro data and in vivo data was determined by Student's $t$ test (2-tailed) and the Mann-Whitney test (2-tailed), respectively. $P<0.05$ was considered to be significant.

Study approval. All mouse experiments were approved by the Institutional Animal Care and Use Committee of The University of Texas M.D. Anderson Cancer Center.

\section{Acknowledgments}

This work was supported in part by U.S. National Cancer Institute grants RO1CA157933, RO1CA152309, R21CA152623, and CA 16672 (Cancer Center Support Grant), and U.S. Department of Defense Breast Cancer Research Program Center of Excellence Award grant W81XWH-06-2-0033. We thank Kathryn L. Hale in M.D. Anderson's Department of Scientific Publications and David M. Wildrick for editing the manuscript. We also thank Jung-Mao Hsu for critically reading the manuscript.

Received for publication May 17, 2013, and accepted in revised form October 18, 2013.

Address correspondence to: Suyun Huang, Department of Neurosurgery-1004, The University of Texas M.D. Anderson Cancer Center, 1515 Holcombe Boulevard, Houston, Texas 77030, USA. Phone: 713.834.6232; Fax: 713.834.6257; E-mail: suhuang@mdanderson.org.
1. Guo X, Wang XF. Signaling cross-talk between TGF- $\beta$ /BMP and other pathways. Cell Res. 2009; 19(1):71-88

2. Moustakas A, Heldin $\mathrm{CH}$. The regulation of TGF $\beta$ signal transduction. Development. 2009; 136(22):3699-3714.

3. Feng XH, Derynck R. Specificity and versatility in TGF- $\beta$ signaling through Smads. Annu Rev Cell Dev Biol. 2005;21:659-693.

4. Massague J. TGF $\beta$ in cancer. Cell. 2008; 134(2):215-230.

5. Chen HB, Rud JG, Lin K, Xu L. Nuclear targeting of transforming growth factor- $\beta$-activated Smad complexes. J Biol Chem. 2005;280(22):21329-21336.

6. Lin X, et al. PPM1A functions as a Smad phosphatase to terminate TGF $\beta$ signaling. Cell. 2006 . 125(5):915-928.

7. Dai F, Lin X, Chang C, Feng XH. Nuclear export of Smad 2 and Smad 3 by RanBP3 facilitates termination of TGF- $\beta$ signaling. Dev Cell. 2009;16(3):345-357.

8. Agricola E, Randall RA, Gaarenstroom T, Dupont S, Hill CS. Recruitment of TIF1 $\gamma$ to chromatin via its PHD finger-bromodomain activates its ubiquitin ligase and transcriptional repressor activities. Mol Cell. 2011;43(1):85-96.

9. Dupont S, et al. FAM/USP9x, a deubiquitinating enzyme essential for TGF $\beta$ signaling, controls Smad4 monoubiquitination. Cell. 2009;136(1):123-135.

10. Tram E, Ibrahim-Zada I, Briollais L, Knight JA, Andrulis IL, Ozcelik H. Identification of germline alterations of the mad homology 2 domain of SMAD3 and SMAD4 from the Ontario site of the breast cancer family registry (CFR). Breast Cancer Res. 2011;13(4):R77.

11. Xie W, et al. Alterations of Smad signaling in human breast carcinoma are associated with poor outcome: a tissue microarray study. Cancer Res. 2002; 62(2):497-505.

12. Koumoundourou D, et al. Prognostic significance of TGF $\beta-1$ and $\mathrm{pSmad} 2 / 3$ in breast cancer patients with T1-2,N0 tumours. Anticancer Res. 2007; $27(4 \mathrm{C}): 2613-2620$.

13. Araki $\mathrm{S}$, et al. TGF- $\beta 1$-induced expression of human $\mathrm{Mdm} 2$ correlates with late-stage metastatic breast cancer. J Clin Invest. 2010;120(1):290-302.

14. Vincent $\mathrm{T}$, et al. A SNAIL1-SMAD3/4 transcriptional repressor complex promotes TGF- $\beta$ mediated epithelial-mesenchymal transition. Nat Cell Biol. 2009;11(8):943-950.

15. Dai M, Al-Odaini AA, Arakelian A, Rabbani SA, Ali S, Lebrun JJ. A novel function for p21Cip1 and acetyltransferase $\mathrm{p} / \mathrm{CAF}$ as critical transcriptional regulators of TGF $\beta$-mediated breast cancer cell migration and invasion. Breast Cancer Res. 2012; 14(5):R127.

16. Park HJ, et al. Deregulation of FoxM1b leads to tumour metastasis. EMBO Mol Med. 2011;3(1):21-34.

17. Kalin TV, Ustiyan V, Kalinichenko VV. Multiple faces of FoxM1 transcription factor: lessons from transgenic mouse models. Cell Cycle. 2011;
10(3):396-405

18. Laoukili J, et al. FoxM1 is required for execution of the mitotic programme and chromosome stability. Nat Cell Biol. 2005;7(2):126-136.

19. Anders L, et al. A systematic screen for CDK4/6 substrates links FOXM1 phosphorylation to senescence suppression in cancer cells. Cancer Cell. 2011;20(5):620-634.

20. Zhang N, et al. FoxM1 promotes beta-catenin nuclear localization and controls Wnt target-gene expression and glioma tumorigenesis. Cancer Cell. 2011;20(4):427-442.

21. Bektas N, et al. Tight correlation between expression of the Forkhead transcription factor FOXM1 and HER2 in human breast cancer. BMC Cancer. 2008;8:42.

22. Carr JR, Park HJ, Wang Z, Kiefer MM, Raychaudhuri P. FoxM1 mediates resistance to herceptin and paclitaxel. Cancer Res. 2010;70(12):5054-5063.

23. Wonsey DR, Follettie MT. Loss of the forkhead transcription factor FoxM1 causes centrosome amplification and mitotic catastrophe. Cancer Res. 2005;65(12):5181-5189.

24. Kwok JM, et al. FOXM1 confers acquired cisplatin resistance in breast cancer cells. Mol Cancer Res. 2010;8(1):24-34.

25. Deheuninck J, Luo K. Ski and SnoN, potent negative regulators of TGF-beta signaling. Cell Res. 2009;19(1):47-57.

26. Wotton D, Lo RS, Lee S, Massague J. A Smad tran- 
scriptional corepressor. Cell. 1999;97(1):29-39.

27. He W, Dorn DC, Erdjument-Bromage H, Tempst P, Moore MA, Massague J. Hematopoiesis controlled by distinct TIF $1 \gamma$ and Smad 4 branches of the TGF $\beta$ pathway. Cell. 2006;125(5):929-941.

28. Chimge NO, et al. Regulation of breast cancer metastasis by Runx2 and estrogen signaling: the role of SNAI2. Breast Cancer Res. 2011;13(6):R127.

29. Côme C, et al. Snail and Slug play distinct roles during breast carcinoma progression. Clin Cancer Res. 2006;12(18):5395-5402.

30. Dhasarathy A, Phadke D, Mav D, Shah RR, Wade PA. The transcription factors Snail and Slug activate the transforming growth factor- $\beta$ signaling pathway in breast cancer. PLoS One. 2011;6(10):e26514.

31. Han G, et al. Distinct mechanisms of TGF-beta1- mediated epithelial-to-mesenchymal transition and metastasis during skin carcinogenesis. J Clin Invest. 2005;115(7):1714-1723.

32. Carr JR, et al. FoxM1 regulates mammary luminal cell fate. Cell Rep. 2012;1(6):715-729.

33. Xue JF, et al. DEDD negatively regulates transforming growth factor- $\beta 1$ signaling by interacting with Smad3. FEBS Lett. 2010;584(14):3028-3034.

34. Petersen M, et al. Smad2 and Smad3 have opposing roles in breast cancer bone metastasis by differentially affecting tumor angiogenesis. Oncogene. 2010;29(9):1351-1361.

35. Kim IM, Ramakrishna S, Gusarova GA, Yoder HM, Costa RH, Kalinichenko VV. The forkhead box $\mathrm{m} 1$ transcription factor is essential for embryonic development of pulmonary vasculature. J Biol
Chem. 2005;280(23):22278-22286.

36. Vincent DF, et al. Inactivation of TIF $1 \gamma$ cooperates with Kras to induce cystic tumors of the pancreas. PLoS Genet. 2009;5(7):e1000575.

37.Xi Q, et al. A poised chromatin platform for TGF- $\beta$ access to master regulators. Cell. 2011; 147(7):1511-1524.

38. Vincent DF, et al. Tif $1 \gamma$ suppresses murine pancreatic tumoral transformation by a Smad4-independent pathway. Am J Pathol. 2012;180(6):2214-2221.

39. Balli $D$, et al. Foxm 1 transcription factor is required for lung fibrosis and epithelial-to-mesenchymal transition. EMBO J. 2013;32(2):231-244.

40. Ikushima H, Miyazono K. TGF $\beta$ signalling: a complex web in cancer progression. Nat Rev Cancer. 2010; 10(6):415-424. 